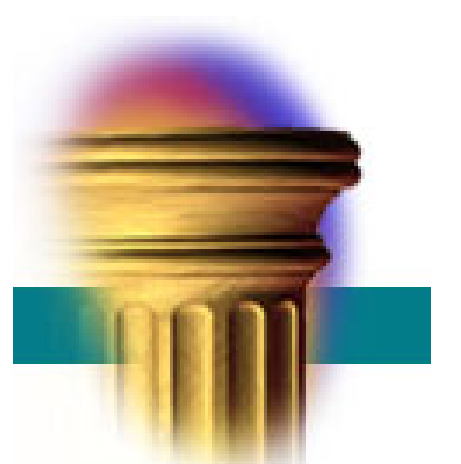

\title{
A Macroeconomic Model of Healthcare So Saturation, Inequality and the ชู Output-Pandemia Tradeoff
}

Enrique G. Mendoza, Eugenio Rojas, Linda L. Tesar, and Jing Zhang

\author{
February 16, 2021
}

\section{WP 2021-02}

https://doi.org/10.21033/wp-2021-02

${ }^{*}$ Working papers are not edited, and all opinions and errors are the responsibility of the author(s). The views expressed do not necessarily reflect the views of the Federal Reserve Bank of Chicago or the Federal Reserve System. 


\title{
A Macroeconomic Model of Healthcare Saturation, Inequality \& the Output-Pandemia Tradeoff*
}

\author{
Enrique G. Mendoza, Eugenio Rojasł, \\ Linda L. Tesar ${ }^{\S}$ and Jing Zhang ${ }^{\mathbb{I}}$
}

February 16, 2021

\begin{abstract}
COVID-19 became a global health emergency when it threatened the catastrophic collapse of health systems as demand for health goods and services and their relative prices surged. Governments responded with lockdowns and increases in transfers. Empirical evidence shows that lockdowns and healthcare saturation contribute to explain the cross-country variation in GDP drops even after controlling for COVID-19 cases and mortality. We explain this output-pandemia tradeoff as resulting from a shock to subsistence health demand that is larger at higher capital utilization in a model with entrepreneurs and workers. The health system moves closer to saturation as the gap between supply and subsistence narrows, which worsens consumption and income inequality. An externality distorts utilization, because firms do not internalize that lower utilization relaxes healthcare saturation. The optimal policy response includes lockdowns and transfers to workers. Quantitatively, strict lockdowns and large transfer hikes can be optimal and yield sizable welfare gains because they prevent a sharp rise in inequality. Welfare and output costs vary in response to small parameter changes or deviations from optimal policies. Weak lockdowns coupled with weak transfers programs are the worst alternative and yet are in line with what several emerging and least developed countries have implemented.
\end{abstract}

${ }^{*}$ The views expressed in this document are those of the authors and not those of the Federal Reserve Bank of Chicago or the Federal Reserve System. We thank participants at the Fifth Annual Conference on International Economics organized by the Federal Reserve Bank of Dallas, University of Houston, and Bank of Mexico for useful comments and suggestions.

${ }^{\dagger}$ University of Pennsylvania and NBER, 133 South 36th Street, Philadelphia, PA 19104, USA. Email: egme@sas.upenn.edu

łUniversity of Florida, 331 Matherly Hall, PO Box 117140, Gainesville, FL 32611, USA. Email: erojasbarros@ufl.edu

§University of Michigan and NBER, 312 Lorch Hall, 611 Tappan Street, Ann Arbor, MI 48109, USA. Email:ltesar@umich.edu

IIFederal Reserve Bank of Chicago, 230 S. LaSalle Street, Chicago, IL, 60604, USA. Email: jing.zhang@chi.frb.org 


\section{Introduction}

A distinguishing feature of the COVID-19 pandemia is that, unlike other viral illnesses that are either more lethal (e.g. Ebola, MERS) or just as contagious but less severe (e.g. Influenza, H1N1), it caused a large, sudden surge in use of human and material resources for prolonged hospitalizations and in demand for medical and cleaning supplies by the economy as a whole. ${ }^{1}$ Thus, in addition to its infection and mortality rates, a key challenge posed by COVID-19 has been the threat of catastrophic collapse of health systems worldwide. The painful experiences of Bergamo, Guayaquil, Mexico City, New York City, Wuhan, and other cities, showed that collapsing health systems prevented hospitals from providing required care to COVID-19 patients and affected the provision of services to those affected by other conditions, both emergencies and elective treatments, thus increasing excess mortality well above the mortality rate of COVID-19 itself.

Governments responded to the threat of collapse of health systems by imposing severe lockdowns that required all non-essential businesses to close and households to obey strict stay-at-home orders, after attempts with weaker social-distancing restrictions failed to slow the spread of the disease. As we document in the next Section, lockdowns have been in place, with some shifting between relaxing and re-tightening, from March, 2020 until the present in several countries. These lockdowns resulted in the largest quarterly declines in GDP in history in many countries during the second quarter of 2020, with a median of -10.6 percent relative to the second quarter of 2019 in a sample with 48 countries (see Section 2 for details). Most countries also implemented policies to provide liquidity to households and firms by increasing transfers and suspending or deferring tax payments. On average, lockdowns have been stricter and transfers programs larger in advanced economies than in emerging and less developed countries. In many cases, these large fiscal interventions produced record-high public deficits and sharp increases in already-high public debt ratios.

The unprecedented economic costs of the lockdowns, on the one hand, and their effectiveness at preventing the collapse of health systems, on the other, pose a critical public policy trade-off: What is the socially-optimal severity of a lockdown that balances the need to contain a pandemia like COVID-19 against its large economic costs? Related to this are other central questions: How does the pandemia affect income and consumption inequality? What is the optimal size of transfers for workers to offset the adverse effects of the lockdown? How does heterogeneity in economic development and health-system strength affect the output-pandemia tradeoff?

This paper answers these questions by proposing a model that deviates from the widely-used approach of integrating the susceptible-infected-recovered (SIR) model of epidemiology into dynamic macroeconomic models. Instead, we propose a framework that focuses on the severe scarcity problem caused by the pandemia and captured by the saturation of health systems and the shortages of health goods. This approach is motivated by the observation that COVID-19 puts health systems at the risk of collapse despite its low mortality and large share of asymptomatic infections. The severe strain on health systems was evidenced by the suspension of regular hospital services to concentrate

\footnotetext{
${ }^{1}$ According to the CDC, the median length of hospitalizations for surviving patients in the U.S. as of October, 2020 was 10 to 13 days. Severe shortages of medical staff, ventilators, N95 masks, disinfectants, and various other health-related products were reported worldwide since the initial outbreak in January 2020.
} 
on COVID treatment and by the sharp increases in occupancy of hospital beds, particular ICU beds, in demand for medical specialists and nurses, and in usage of critical equipment such as respiratory ventilators (see Section 2 for details). As a result, excess mortality rates rose significantly above those explained by COVID. For instance, while COVID's infection fatality rate is estimated at 0.65 percent (according to the CDC), excess weekly deaths as a percent of expected deaths between March and June, 2020, peaked at 154 percent in Spain, 108 in the United Kingdom, 90 in Italy, and 45 in the United States, and in Mexico City excess mortality reached 300 percent in the March-May period. ${ }^{2}$

The paper starts with an empirical examination of cross-country data that documents the effects of COVID-19 on resource scarcity and relative prices for health goods and services, excess mortality rates and severity of lockdowns. In addition, we conduct an empirical analysis showing that a nontrivial share of cross-country differences in observed output declines caused by the pandemia is explained by variables that proxy for the severity of lockdowns, resource shortages and pre-COVID19 health system strength, even after controlling for COVID-19 cases and fatalities.

In the model, the pandemia arrives as a large, temporary shock to the subsistence demand for health goods and services in a Stone-Geary utility function. The shock is larger at higher capital utilization rates. The degree of saturation of the health sector is represented by the gap between the available supply of health goods and services and their subsistence level. The catastrophic (i.e., nonlinear) nature of a health-system collapse is captured by the Inada condition of Stone-Geary preferences. The tradeoff with economic activity works through the dependency of the subsistence demand for health on utilization. Lower utilization relaxes the capacity of the health system, moving it away from its saturation point, but it implies reduced demand for factors of production, reduced output and lower factor payments in the non-health sector. This also introduces a "utilization externality," because firms do not internalize the link between utilization and health-system-saturation when choosing utilization. The size of the hike on subsistence demand for health and the severity of the externality depend on the elasticity of the subsistence health demand to utilized capital. A planner who takes the externality into account has a social marginal cost of utilization higher than the private cost when a pandemia is active. This results in a socially-optimal reduction in utilization during a pandemia, which is decentralized as a competitive equilibrium by mandating an optimal lockdown (i.e., a binding constraint on utilization tighter than the technologically feasible limit).

In order to study the implications of the output-pandemia tradeoff for inequality and the design of liquidity-provision programs, the model includes two types of agents: entrepreneurs, who collect wages and all capital income from the health and non-health sectors, and workers, who collect only wage income. We show that, as the pandemia causes health-system saturation to worsen and the relative price of health goods to rise, inequality in terms of both relative income and relative excess consumption (or relative marginal utilities) of entrepreneurs vis-a-vis workers worsens. As a result, the optimal policy calls for increased transfers to workers. ${ }^{3}$ Hence, the optimal policy response to a pandemia includes both a lockdown and higher transfers.

\footnotetext{
${ }^{2}$ See https://ourworldindata.org/excess-mortality-covid and https://www.washingtonpost.com/world/the_americas / mexico-citycoronavirus- excess-death-toll/2020/07/02/2baaab3e-bbbb-11ea-80b9-40ece9a701dc_story.html.

${ }^{3}$ Inequality makes transfers desirable even without a pandemia for a planner who is utilitarian or weights workers by more than their share of the population. Still, optimal transfers rise with a pandemia because inequality worsens.
} 
In the model, aggregate allocations and prices are independent of individual allocations in both the decentralized and social planner's equilibria. In particular, the effects of the pandemia on utilization and the relative price of health goods are unaffected by agent heterogeneity, inequality and the planner's welfare weights. The effects of the pandemia on inequality and the optimal transfers, however, do depend on the severity of the utilization externality. The stronger the externality, the closer to saturation the pandemia brings the health system and the higher the increase in the price of health goods. This worsens income and excess consumption inequality, since the income of workers falls more and their consumption moves closer and faster to their subsistence levels, which makes larger transfers optimal. The size of the optimal transfers also depends on the planner's welfare weights and the fraction of workers relative to entrepreneurs (i.e., the pre-pandemia wealth distribution).

We explore the model's quantitative implications by examining numerical solutions based on a calibration to U.S. data. Key to this calibration are the determination of the subsistence demand for health in "normal times" (i.e. without a pandemia) and the parameterization of the function that drives the jump in this subsistence demand when the pandemia hits. We determine the former by estimating a standard linear-expenditure-system regression using U.S. data. For the latter, we use a linear function that simplifies the calibration into choosing the value of the elasticity of subsistence health demand with respect to utilized capital. Since little is known about this elasticity, we study results for the interval of values for which the competitive equilibrium with pandemia exists ( 0 to 0.107). Because of the Inada condition of the Stone-Geary utility, there is an upper bound of the elasticity at which workers' health consumption equals subsistence demand and hence there is no competitive equilibrium solution. For the weights of the planner's social welfare function, we focus on the case in which the planner's solution supports the competitive equilibrium in normal times.

Among the feasible elasticities, we study a particular scenario in which the planner's optimal lockdown yields an output drop equal to the drop in U.S. non-health GDP in the second quarter of 2020 (an elasticity of about 0.09 ). This scenario rationalizes the observed non-health output drop as resulting from an optimal lockdown that reduces utilization by 15 percentage points. The optimal increase in transfers equals 10.9 percentage points of GDP. This planner's equilibrium is compared with two competitive equilibria computed using the same elasticity: A "no lockdown" (NL) case in which policy is unchanged and an "observed lockdown" (OL) case in which the optimal lockdown is imposed in ad-hoc fashion. In the OL case, utilization and the other aggregate variables match the planner's but transfers are unchanged. This scenario shows the implications of implementing the optimal lockdown without the optimal transfers.

Assuming a pandemia that lasts four quarters, the optimal policies yield welfare gains of 0.82 and 0.33 percent $\mathrm{v}$. the $\mathrm{NL}$ and OL cases, respectively (in terms of the standard welfare measure given by a compensating variation in consumption constant across time that equalizes lifetime utility under alternative regimes). Hence, a policy of implementing a lockdown as severe as the optimal one without increasing transfers yields a welfare gain of 0.49 percent relative to unchanged policies but it still means a loss of 0.33 percent relative to the optimal policy regime. This indicates that the adverse effects of the pandemia on inequality are large. Inequality is at its worst in the NL case, for which the ratio of excess consumption of entrepreneurs to workers rises to 16.5 during the pandemia, 4.75 times 
higher than under the optimal policies (which by construction keep the ratio at its normal-times level of 3.5). In the OL case the ratio still rises to 9.5, 2.75 times larger than the optimal ratio. The same pattern affects income inequality: it worsens more in the NL than the OL case, because the relative price of health goods rises 158 percent in the former v. 101 in the latter. Thus, the entrepreneur's capital income from the health sector rises much more in the NL than the OL case. Increasing the elasticity of subsistence health demand to utilized capital above 0.09 yields much larger welfare gains and transfers, with the former growing infinitely large as the pandemia moves workers closer to their subsistence demand and at a much faster pace than for entrepreneurs. The relevance of inequality is also reflected in a comparison of these results with those for a representative-agent version of the model. This economy yields sharply smaller welfare gains for the same elasticities of subsistence health demand to utilized capital.

The results for the full interval of feasible elasticities of subsistence health demand to utilized capital show that pandemias start to have sizable effects on macro aggregates and inequality at elasticities above 0.05 . The output-pandemia tradeoff produces concave, inverse relationships between the planner's optimal utilization (or non-health output) and that elasticity. Relative prices and excess consumption ratios in the NL and OL solutions, as well as the welfare gains under the optimal policies, are increasing and convex functions of the elasticity. Hence, small errors in measuring the elasticity result in non-trivial differences in the size of optimal lockdown and transfer policies and their effects on aggregate variables and inequality.

The planner neutralizes the strong adverse impact of the pandemia on consumption and income inequality as a result of the direct effect of higher transfers and the indirect effect of the lockdown. The latter reduces inequality because it mitigates the relative price hike and the rise in the excess consumption ratio. Preventing inequality from worsening contributes over 90 percent of the welfare gains of the optimal policies for all elasticities of subsistence health demand to utilized capital that produce non-negligible pandemias. The aggregate effect of the lockdown removing the utilization externality accounts for the remaining 10 percent. Inequality and distributional effects also makes the model more plausible. A planner in a representative-agent version of the model only gains by removing the utilization externality and as a result it needs larger elasticities of subsistence health demand to utilized capital (of at least 0.13 ) in order to yield non-trivial welfare gains. These elasticities, however, imply output drops much larger than those that have been observed.

We also conduct an analysis of the implications of deviating from the optimal policies for a large set of lockdown and transfer policy pairs. These deviations result in sizable welfare costs relative to the optimal policies but still policy intervention to respond to the pandemia is preferable to no intervention. Moreover, transfers and lockdowns are substitutable to a degree and using either tool with sufficient strength can get reasonably close to the gains attained by the optimal policies. The reason is that either a large increase in transfers or a strict lockdown weakens significantly the strong adverse effects of the pandemia on inequality. For the same reason, however, combining weak lockdowns with small transfers programs is the worst policy choice. Unfortunately, this seems to be what is occurring in emerging and least developed countries, which on average responded to COVID-19 with weaker lockdowns and smaller fiscal interventions than advanced economies. Using the data 
from Section 2 and from the IMF's Fiscal Monitor, we found that income per capita has a correlation with COVID lockdown effectiveness of roughly -0.2 but its correlation with Covid-related transfers is 0.5 . Through September 2020, advanced economies increased transfers by nearly 10 percentage points of GDP on average while the increases in emerging and least developed countries averaged 4.4 and 3 percentage points, respectively.

The model and the quantitative findings also have important implications for the analysis of cross-country or cross-region responses to COVID-19. The model predicts that the pandemia has been more damaging for countries with higher wealth inequality and/or weaker pre-pandemia conditions in characteristics such as quality or capacity of health systems, income per capita, etc. Weaker pre-pandemia conditions can be thought of as implying higher elasticities of subsistence health demand to utilized capital which the model associates with larger optimal lockdowns and output drops. The relative size of the health sector also reflect cross-country differences in health systems. For a given elasticity, the model predicts larger effects of pandemias in countries with smaller health sectors or smaller shares of non-health expenditures.

This paper is related to the growing COVID-19 macro literature. Most of this literature emphasizes the probabilistic dynamics of contagion, infection and death (or recovery) from the disease itself, by incorporating them into macro models using the canonical SIR/SEIR models from epidemiology. The contribution of our work is the focus on resource scarcity and the saturation of the health sector as the drivers of the output-pandemia tradeoff and its distributional implications. ${ }^{4}$ In SIR/SEIR-based models, decentralized equilibria are inefficient because the planner internalizes these disease dynamics and the social welfare function depends negatively (positively) on the death (recovery) rates. In contrast, in the model proposed here social welfare is a standard aggregation of individual preferences over consumption and labor, and the adverse implications of a pandemia for efficiency and inequality result from the surge in subsistence demand for health that it causes, which is larger at higher utilization and affects workers more severely than entrepreneurs. Moreover, this framework also accounts for large increases in the relative price of health goods during a pandemia.

Alvarez et al. (2020), Atkeson (2020) and Eichenbaum et al. (2020) initiated the literature on quantitative SIR-based macro models. In these models, the pandemia affects macroeconomic outcomes through demand and supply effects. Infections and mortality increase with consumption and hours worked. Sick workers become less productive or work less and consume less, and consumption and labor have feedback effects on infections. In addition, contagion causes externalities as agents do not internalize how their individual actions affect the SIR dynamics. Lockdowns improve efficiency by tackling this externality. Alvarez et al. (2020), Favero et al. (2020) and Jones et al. (2020) introduce also a congestion externality by modeling the COVID-19 fatality rate as an increasing function of total infections above a constant rate. This externality is similar to the utilization externality resulting from the adverse effect of utilization on the health subsistence demand in our model, but it differs in that in the SIR models congestion increases fatalities, which the planner is

\footnotetext{
${ }^{4}$ This is in parallel with the public health literature on pandemias, in which a branch focusing on resource scarcity and saturation of hospitals (e.g. Ajao et al., 2015, Halpern and Tan, 2020) coexists with the SIR/SEIR epidemiology branch (see the survey by Britton, 2010).
} 
assumed to dislike. Hence, although both models predict that lockdowns are desirable because of health-system congestion, the mechanism driving the result is different. In particular, in our setup lockdowns are desirable because the pandemia brings all agents closer to their subsistence level of health regardless of whether they are infected and of the COVID-19 fatality rate, and redistribution is desirable because this effect hits workers more severely than entrepreneurs.

The macro-SIR/SEIR framework has also been used in models with agent and sectoral heterogeneity, as in the studies by Acemoglu et al. (2020), Baqaee et al. (2020), Bodenstein et al. (2020), Azzimonti et al. (2020), Glover et al. (2020), Guerrieri et al. (2020), Hur (2020), Kaplan et al. (2020), Krueger et al. (2020) and Rampini (2020). These studies suggest that lockdowns should be targeted differentially across sectors, with their severity depending on how contact-intensive sectors are, the composition of workers in the sector (age, susceptibility, health), how essential and easy to substitute are the goods produced by the sector, and how connected agents are in a production network. In most of these articles, agent and/or sectoral heterogeneity drive the policies due to their effect on aggregate outcomes and on the dynamics of infection, recovery and death rates.

SIR models with wealth and income inequality have also been used to study the optimal redistributive policy during a pandemia. Glover et al. (2020) find that the optimal policy involves redistribution from agents that continue working towards those who cannot or who lost their jobs. Bloom et al. (2020) argue that lockdowns and transfers should consider dimensions of income and wealth inequality, because low-income or low-wealth workers typically are more affected by lockdowns since their occupations are less suitable for teleworking (see also Galasso, 2020, Mongey et al., 2020 and Palomino et al., 2020). Thus, economies with a larger fraction of low-wealth agents require milder lockdowns and/or larger transfers. Chetty et al. (2020) examine heterogeneous effects on consumption. Using high-frequency data, they find that COVID-19 has had negative effects on consumption, with lower-income agents being affected disproportionately.

The SIR framework has also been used in open economy models. Arellano et al. (2020) embed SIR dynamics into an Eaton-Gersovitz sovereign default model. The sovereign cares about the fatality rate and can impose lockdowns in order to mitigate the magnitude of the health crisis. Since lockdowns depress output, the sovereign has the incentive to borrow abroad to smooth consumption, but this increases default risk and hence limits the planner's ability to impose aggressive lockdowns as its borrowing capacity is restricted, costing additional lives. Cakmakli et al. (2020) study a multi-sector model with sectoral supply and demand shocks that vary with infections depending on lockdowns. The openness of the economy matters via external demand shocks and input-output linkages.

There are other influential macro models of COVID-19 that do not use the SIR setup and consider the role of financial frictions on firms. Gourinchas et al. (2020) study effects on small and medium enterprises using a model in which the virus causes labor supply constraints that vary by sector and also causes sectoral and aggregate demand shocks and business failures. They find that firm bailouts are better than labor subsidies for reducing bankruptcies and saving jobs, and that targeted bailouts have sizable benefits at lower GDP costs. Céspedes et al. (2020) and Fornaro and Wolf (2020) show that financial frictions combined with a negative productivity shock during the pandemia can 
produce equilibria with long-lasting crises and slow recoveries. Elenev et al. (2020) study a setup in which firms can go bankrupt due to the pandemia, and study how bailouts can help save firms that are experiencing financial distress. Faria e Castro (2020) models the pandemia as a shutdown of the contact-intensive services sector (caused by a utility shock) that is transmitted to other sectors in the economy, while Guerrieri et al. (2020) model it as a shock on the labor supply of a productive sector that requires physical interactions (a fraction of workers becomes unable to work in this sector). In turn, reduced consumption of goods from this sector reduces the households' health. These studies find that transfer payments to workers in sectors affected by the pandemia are socially optimal.

The rest of the paper is organized as follows. Section 2 provides empirical evidence on the relevance of healthcare saturation and important empirical regularities of the macro effects of the COVID-19 pandemia. Section 3 describes the model. Section 4 presents the quantitative results of the calibrated model. Section 5 provides some conclusions.

\section{Empirical Evidence}

In this Section, we review the empirical evidence on COVID-19 that motivates the theoretical model. The discussion is divided into four parts: 1) a review of the resource shortages and constraints on medical systems for managing the pandemia, 2) the impact of the pandemia on prices of critical medical services and equipment, 3) international evidence on the severity and duration of lockdowns, and 4) a cross-country analysis of the determinants of output collapse during the pandemia.

\subsection{Resource shortages and capacity constraints for COVID-19}

Saturation of the health system caused by COVID-19 has three important components. The first is the capacity of hospitals to treat COVID patients, particularly to provide them with ventilation therapy. The second is the closure of non-Covid related medical and hospital services, as hospitals are dedicated to COVID patients and medical practices and elective procedures are shut down. The third are the shortages of medical and cleaning supplies as the healthcare and non-healthcare sectors as well as households aim to build up subsistence inventories.

Consider first hospital capacity to treat patients. Evidence from COVID-19 projections and existing studies from the public health literature shows that pandemias pose a serious risk to cause health systems to collapse. On March 26, 2020, the Institute for Health Metrics and Evaluation (IHME) of the University of Washington issued a forecast of the likely stresses on the U.S. medical system due to COVID-19. Their analysis, based on a state-by-state assessment of medical facilities, warned that in the absence of large-scale public health interventions, particularly mitigation measures (i.e. lockdowns) the demand for intensive care facilities would outstrip existing supply in a matter of days.

IHME's analysis focused on ICU beds, but health systems can collapse well before running out of regular and ICU hospital beds as they run out of medical specialists, nursing staff, equipment and materials needed to treat patients in respiratory distress. Ajao et al. (2015) assessed the capacity of the U.S. healthcare system to respond to increased demand for ventilation therapy due to a hypothet- 
ical influenza pandemic outbreak under three levels of stress on the health system: (i) conventional capacity (usual and normal patient care); (ii) contingency capacity (minor adaptation of treatment approaches) and (iii) crisis capacity (fundamental, systematic change in which standards of care are significantly altered to allow treatment of a greater number of patients). Their study identified four key components necessary to provide ventilation therapy:

1. Supplies, such as ventilators, ancillary supplies, and equipment.

2. Space, namely hospital beds equipped for ventilation and critical care.

3. Staff, consisting of specialized medical personnel to manage patients on ventilators.

4. Systems, namely accessible, exercised plans to rapidly increase ventilation therapy capacity.

Hence, the provision of ventilation therapy is akin to a Leontief technology that requires complementary inputs in relatively fixed proportions. As a result, hitting a constraint on one input limits the ability to provide ventilation therapy. Taking as given the estimated number of ventilators availabe in 2010 and assuming that they would not be the constraining factor, Ajao et al. (2015) showed that at the peak of the hypothetical influenza pandemia in the United States, the constraining factor for ventilation therapy in scenario (iii) would be the number of respiratory therapists, not the number of beds. The maximum number of additional patients that could be put in a ventilator would range from 56,300 to 135,000 , which would fall short of the number of available beds enabled for ventilation therapy. In fact, 32,300 to 42,300 beds would go unused.

Halpern and Tan (2020) assess U.S. capacity for treating COVID-19 patients under current conditions. Based on surveys of U.S. hospitals, they report that acute care hospitals own 62,188 fullfeatured mechanical ventilators. Adding other equipment that can be diverted to ventilator use (e.g. from operating rooms and the U.S. stockpile) has the potential to bring the total up to 200,000 devices nationally. Recent projections suggest that approximately 960,000 patients in the US would require ICU ventilatory support, though not all patients would be treated at the same time. But even if the number of patients could be optimally staggered, they conclude the critical factor is staffing. According to the BLS there are approximately 130,000 respiratory therapists in the labor force. However, there are far fewer respiratory intensivists, physicians certified to provide care for critically ill patients. The American Hospital Association estimates that there are roughly 29,000 intensivists nationwide, and about half of acute care hospitals have no intensivists on their staff. Halpern and Tan (2020) conclude, "At forecasted crisis levels, we estimate that the projected shortages of intensivists, critical care APPs, critical care nurses, pharmacists, and respiratory therapists trained in mechanical ventilation would limit the care of critically ill ventilated patients" (p. 1). "Moreover, even in the 50 percent of acute care hospitals with intensivists, the intensivist team may be overstretched as new ICU sites are created or experienced ICU staff become ill" (p. 8).

Li et al. (2020) apply the dynamics of the COVID-19 outbreak in Wuhan to the United States and reached similar conclusions as Ajao et al. (2015) and Halpern and Tan (2020). In their analysis, "the projected number of prevalent critically ill patients at the peak of a Wuhan-like outbreak in US cities was estimated to range from 2.2 to 4.4 per 10,000 adults, depending on differences in age distribution and comorbidity (ie, hypertension) prevalence" (p. 1). Based on a population of roughly 210 million 
adults, this is an afflicted population of 460,000 to 920,000. "[I]f a Wuhan-like outbreak were to take place in a US city, even with social distancing and contact tracing protocols as strict as the Wuhan lockdown, hospitalization and ICU needs from COVID-19 patients alone may exceed current capacity... Plans are urgently needed to mitigate the consequences of COVID-19 outbreaks on local health care systems in US cities" (pp. 5-7).

The second aspect of health system saturation caused by COVID-19 is evidenced by the suspension or drastic reduction in provision of non-Covid-related medical services and treatments. Hospitals expanded capacity to treat COVID patients as envisaged in the critical scenario (iii) of Ajao et al. (2015), by reallocating physical and human resources normally dedicated to other uses to treat COVID patients. In addition, in many instances lockdowns implied closure of medical and dental practices, laboratories, and outpatient surgery facilities. These changes and restrictions caused a sharp increase in mortality, as measured by the standard excess mortality P-Score. We collected cross-country data for P-cores computed using the number of total deaths, COVID- and non-COVIDrelated, at a weekly frequency minus the average of deaths over the 2015-2019 period and divided by the same 2015-2019 average (the source was https:/ /ourworldindata.org/excess-mortality-covid and some country-specific sources). Table 1 shows the highest weekly P-Scores for the January-June, 2020 period in 35 countries. The mean (median) reached 42.9 (23.8) percent, but in several cases it exceeded 50 percent (Belgium, Chile, Italy, Netherlands, Mexico, Peru, Spain, Turkey and the U.K.). Since P-scores combine COVID and non-COVID fatalities, they are a noisy measure of fatalities not caused directly by the disease, but in the analysis of cross-country output drops conducted below we will control for COVID fatalities to identify the effect of non-COVID excess mortality.

The third element of resource shortages due to COVID-19 relate to health goods and services and cleaning supplies for the economy as whole. We document the impact of these shortages by examining the evolution of the relative prices of the affected goods and services in the next subsection.

\subsection{Rising prices of PPE and medical equipment}

COVID-19 caused severe shortages of medical equipment and cleaning supplies that resulted in sharp price hikes. In the United States, spikes in prices for cleaning supplies, toilet paper and medical masks prompted consumer groups to complain of price gouging. Several states embarked on campaigns inviting consumers to send images of purported price gouging. A Google search of images of "COVID price gouging" yields pictures of $8 \mathrm{oz}$. bottles of hand sanitizer priced at $\$ 50$ and Clorox wipes at over $\$ 40$ per container. The U.S. PIRG consumer watchdog association reported inflation rates of COVID-19 related goods ranging from 200 percent for thermometers to 1,300 percent for anti-bacterial handwipes. These goods disappeared from store shelves where they were priced at regular prices, resulting in massive, prolonged stockouts of key items in retail chains. The prices of these goods facing severe shortages are mismeasured in aggregate price indexes, because these indexes rely on surveys of posted retail prices even for out-of-stock goods (e.g. on August 18, 2020, the posted but out-of-stock price for Clorox disinfecting wipes 75ct was $\$ 6.59$ on the CVS website, but they were available on eBay for \$29 plus \$11.67 shipping; Lysol disinfectant spray 19oz was $\$ 5.97$ 
at Home Depot but out of stock, while on eBay it was available for $\$ 12.50$ plus $\$ 17.50$ shipping).

Table 1: Excess Mortality P-Scores, Percent

\begin{tabular}{lrlr}
\hline \hline Country & P-score & Country & P-score \\
\hline Peru & 163 & Israel & 20.3 \\
Spain & 154.5 & South Africa & 18 \\
United Kingdom & 108 & Colombia & 18 \\
Belgium & 104.4 & Greece & 16 \\
Italy & 96.8 & Austria & 16 \\
Mexico & 87 & Germany & 14.5 \\
Netherlands & 74.9 & Russia & 14 \\
Chile & 68.7 & Finland & 13.6 \\
France & 65.2 & Ireland & 12 \\
Turkey & 54 & Australia & 12 \\
Indonesia & 50 & New Zealand & 11.9 \\
Sweden & 47.1 & Denmark & 10.5 \\
United States & 44.9 & South Korea & 9.6 \\
Switzerland & 44 & Norway & 9.5 \\
Brazil & 42 & Taiwan & 9.2 \\
Canada & 26.2 & Czech Republic & 8.9 \\
India & 25 & Poland & 8.3 \\
Portugal & 23.8 & & \\
\hline \hline
\end{tabular}

\begin{abstract}
Notes: The scores shown are the maximum of weekly P-scores over the January-June, 2020 period computed as the number of total deaths in each week minus the average of deaths over the 2015-2019 period and divided by the same 2015-2019 average. For most countries, weekly P-scores were retrieved from https://ourworldindata.org/excess-mortality-covid on 12/2/2020. Data for Brazil, Indonesia, Mexico, Peru, Russia, South Africa, and Turkey are from https://www.economist.com/graphic-detail/2020/07/15/trackingcovid-19-excess-deaths-across-countries, for Colombia, India and Ireland from https://www.nytimes.com/interactive/2020/04/21/world/coronavirus-missingdeaths.html, and for Australia from the Australian Bureau of Statistics. Data for Indonesia and Turkey cover only Jakarta and Istanbul, respectively.
\end{abstract}

Similar price dynamics were observed for medical equipment as hospitals and even state governments competed for the limited supply of PPE and ventilators. On April 24, 2020, National Public Radio aired a report entitled "Are Illinois Officials Paying Hugely Inflated Prices For Medical Supplies?" A government audit revealed spending up to \$174 million on COVID-related medical supplies and equipment, including \$13 million for 200 ventilators, a 100 percent markup over the pre-COVID price. The governor stated that "A typical ventilator that's useful in an [intensive care unit ] situation, the price starts at $\$ 25,000$, maybe up to $\$ 35,000$ or $\$ 40,000, \ldots$ When we're paying more than that, that's typically because the market has bid up the prices for any available ventilators. Let me be clear: There are very few ventilators available in the entire world. We are acquiring whatever we can so that we're ready in the event there's a spike in ICU beds and a need for ventilators..."

Wages for travel nurses responded to the increased demand for hospital staff. During peak COVID periods in the Spring and Summer of 2020, the weekly compensation rate for travel nurses roughly doubled, according to the Health IT website (HIT.net). The "Travel Nurse Compensation 
Report" data from BLS show more modest salary increases for nurses in early 2020 of less than five percent, but these are somewhat misleading, however, as they aggregate the salaries of specialists in fields where medical services actually declined (e.g. voluntary medical care, private medical practices) along with the rising salaries of nurses that are ICU and respiratory specialists.

Table 2 shows evidence of the large price hikes caused by Covid-related shortages of essential health goods and services. The median price increase for transactions of thirteen key goods, including N95 masks, ventilators, thermometers and disinfectants, reached 259 percent from March to April, 2020. The Table also shows inflation rates for aggregate price indexes. Health-services prices rose at annualized rates ranging from 3.1 to 4.7 percent in the second quarter of 2020, while the price index for private goods-producing industries fell -16.1 percent. ${ }^{5}$ Hence, prices of health services relative to those for goods-producing industries rose between 19.2 to 20.8 percent, and for the specific health goods listed in the Table, the median relative price hike exceeded 275 percent.

\subsection{Duration and severity of lockdowns}

Figure 1 illustrates the severity and duration of the lockdowns implemented in a group of sixteen advanced and emerging economies. The data correspond to the Government Response Stringency Index constructed as part of the Oxford COVID-19 Government Response Tracker (OxCGRT). ${ }^{6}$ This index combines information from nine indicators including school and business closures, and travel bans in a scale from 0 to 100 (with 100 for the strictest). In countries where policies vary within the country, the index corresponds to the strictest area. The index is available for 180 countries.

The Figure shows that strict lockdowns were implemented in all countries by mid March, 2020. In most cases, the index peaked around 75-80 percent, except in Sweden, well-known for its less restrictive stance. Even in Sweden, however, the stringency index reached nearly 50 percent. Moreover, lockdowns have persisted from March to the latest available data as of the date of this paper. The severity of the lockdowns has fluctuated somewhat and in several cases declined (in some like France and New Zealand quite sharply), but as of the Dec. 2020 data all countries still maintained significant restrictions on economic activity relative to the pre-COVID-19 status. Even in Sweden, the stringency index fell slightly from its peak but it remains above 30 percent.

\subsection{Economic activity}

The strict lockdowns caused deep recessions, in many cases the largest recorded declines in quarterly GDP. Figure 2 shows year-on-year drops in GDP in the second quarter of 2020 for 48 advanced and emerging economies. ${ }^{7}$ The mean (median) drop was a staggering -11.5 (-10.6) percent.

\footnotetext{
${ }^{5}$ Health services at this level of aggregation include some for which prices fell as a result of suspension of elective treatments, routine medical, dental and optical appointments, etc.

${ }^{6}$ Available at https://www.bsg.ox.ac.uk/research/research-projects/coronavirus-government-response-tracker

${ }^{7}$ Most of the data are from ourworldindata.org, fred.stlouisfed.org, and www.focus-economics.com. For China and Hong Kong, the Figure shows the GDP drop in the first quarter, because these countries entered the pandemia earlier.
} 
Table 2: Price Changes of Key Health Goods \& Services During the COVID-19 Pandemia

\begin{tabular}{|c|c|c|}
\hline Item & Price Change & Source \\
\hline N95 Masks & $1513 \%$ & SHOPP \\
\hline 3M N95 Masks & $6136 \%$ & SHOPP \\
\hline Hand Sanitizer & $215 \%$ & SHOPP \\
\hline Isolation Gowns & $2000 \%$ & SHOPP \\
\hline Face Shields & $900 \%$ & SHOPP \\
\hline Soap & $184 \%$ & SHOPP \\
\hline Ventilators & $80 \%$ & NY State \\
\hline Clorox Disinfecting Wipes & $660 \%$ & US PCW \\
\hline Anti-Viral Facial Tissues & $254 \%$ & US PCW \\
\hline Bleach Cleaner & $238 \%$ & US PCW \\
\hline Thermometers & $200 \%$ & US PCW \\
\hline Face Masks & $259 \%$ & US PCW \\
\hline Anti-Bacterial Hand Wipes & $1294 \%$ & US PCW \\
\hline \multicolumn{3}{|c|}{ Aggregate Price Indexes (Q2:2020 v. Q1:2020 annualized) } \\
\hline Physicians' Services & $4.68 \%$ & BLS \\
\hline Medical Care Services & $4.40 \%$ & BLS \\
\hline Hospital Services & $3.14 \%$ & BLS \\
\hline Health Care and Social Assistance & $3.60 \%$ & BEA \\
\hline Private Goods-Producing Industries & $-16.10 \%$ & BEA \\
\hline
\end{tabular}

\begin{abstract}
Notes: Personal protective equipment price changes are for April 2020 relative to pre COVID-19 levels (not annualized) reported by the Society for Healthcare Organization Procurement Professionals (SHOPP). Price changes (not annualized) for items reported by the US PCW (US PIRG Consumer Watchdog) correspond to the difference between the price listed in Amazon and the lowest price listed by other platforms during August 2020. BLS and BEA price indexes correspond to percent changes between 1st and 2nd quarter of 2020, annualized. Private goods-producing industries are: agriculture, forestry, fishing, and hunting; mining; construction; and manufacturing.
\end{abstract}

How much of the output declines was due to healthcare system saturation? To answer this question, we test for the relative importance of lockdowns, COVID case and mortality rates, and measures of the stress on healthcare systems in explaining cross-country differences in the magnitude of the decline in GDP.

The severity of lockdowns can be gauged with de-jure or de-facto measures. For the former, we use again the Oxford Stringency Index, and for the latter we use the components of the Google COVID-19 Community Mobility Reports that track movement to and from retail, recreational and work places. Mobility is reported as percent change relative to a pre-COVID baseline (the median for the five-week period Jan. 3rd-Feb. 6, 2020). ${ }^{8}$ This indicator is useful because it captures the actual mobility of the population while the stringency index captures legal restrictions.

Unconditional scatter diagrams of both de-jure and de-facto lockdown severity indicators show a clear relationship with the observed quarterly GDP drops (see Figure 3). Output drops were larger in countries with stricter lockdowns, whether measured by a higher stringency index or lower community mobility. These scatter diagrams only tell part of the story, however, because other variables

\footnotetext{
${ }^{8}$ For both the Stringency Index and Community Mobility, we use 30-day averages as of the end of November, which were obtained from the components of Bloomberg's Covid Resilience Index, see https://www.bloomberg.com/news/articles/2020-11-24/inside-bloomberg-s-covid-resilience-ranking.
} 
are likely to jointly affect economic activity and lockdowns, and we are interested in particular in determining whether variables that proxy for resource shortages and capacity constraints (i.e., health system saturation) play a role. To identify those effects, we conduct a panel regression analysis in a cross-section of 35 countries.

Figure 1: Lockdown Stringency Index for Selected Countries
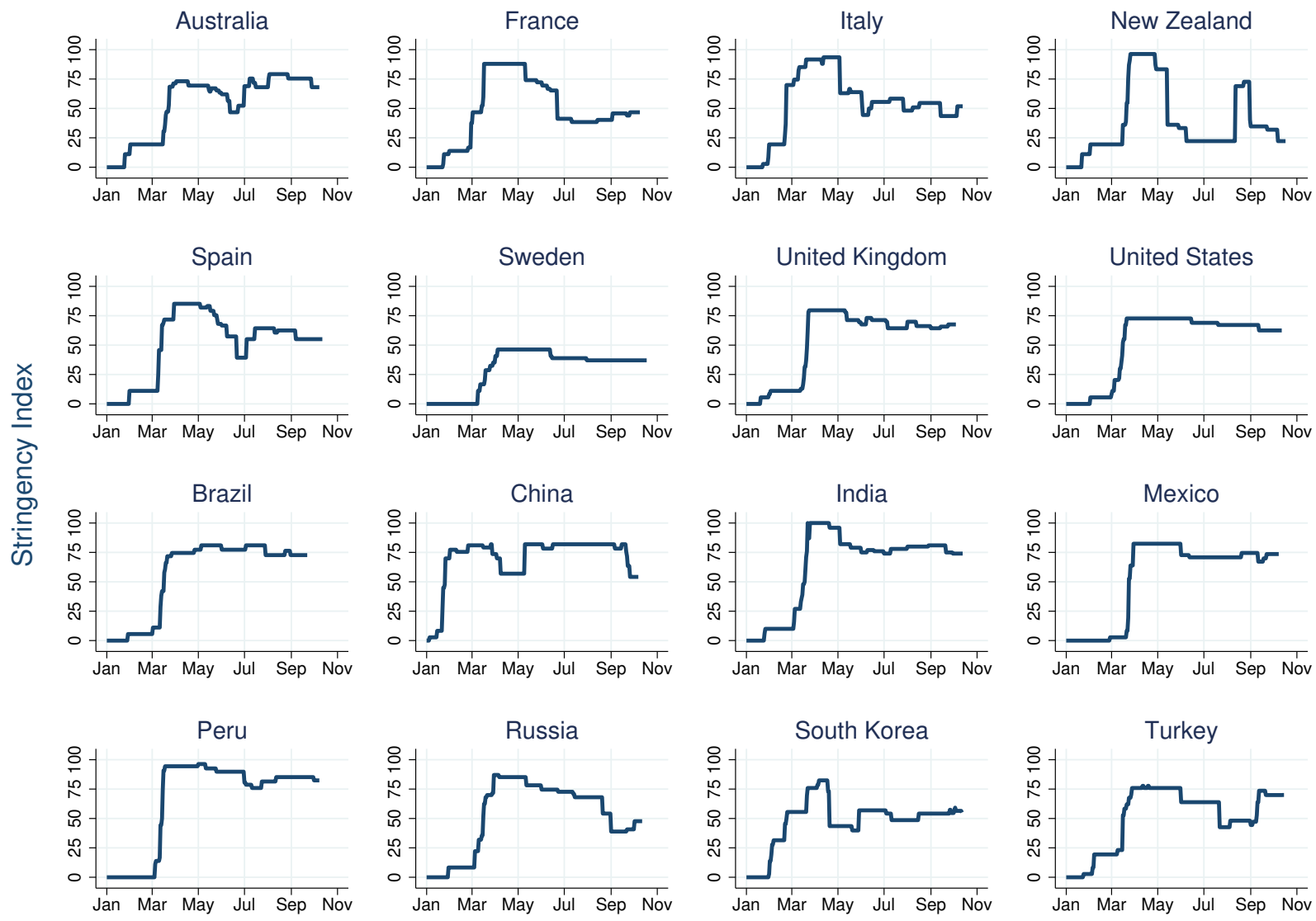

Source: Government Response Stringency Index available from the Oxford COVID-19 Government Response Tracker.

The dependent variable in this analysis is the contraction in GDP, as measured by the fall in the second quarter of 2020 relative to the same quarter in 2019. The independent variables include: the stringency index and community mobility changes described above, two variables to capture the infection and mortality rates of COVID-19 itself (COVID cases for November, 2020 and cumulative COVID deaths through the end of November), and four variables as proxies for health system resources and capacity limits. The latter include a proxy for the non-COVID excess mortality rate (defined as the residual from regressing the excess mortality P-scores on COVID deaths), hospital beds, the log of 2019 GDP per capita, and the UNDP's human development index (HDI) that combines life expectancy, educational attainment and gross national income per capita. COVID cases and fatalities and hospital beds are in units per one-million inhabitants and the rest of the variables are in percent. The data are available for 48 countries for most variables, but excess mortality P-scores are only available for 35 , which sets the sample size of the regressions. The variables are expressed 
in deviations from their means and the regressions are estimated using MM Robust Least Squares. ${ }^{9}$ The coefficients for all variables but hospital beds and COVID cases and deaths are elasticities, since the data are all in percent, and hence they are comparable. Coefficients for hospital beds and COVID cases and deaths are comparable, because the data for each are per one-million inhabitants.

Figure 2: Year-on-Year 2020:Q2 GDP Declines

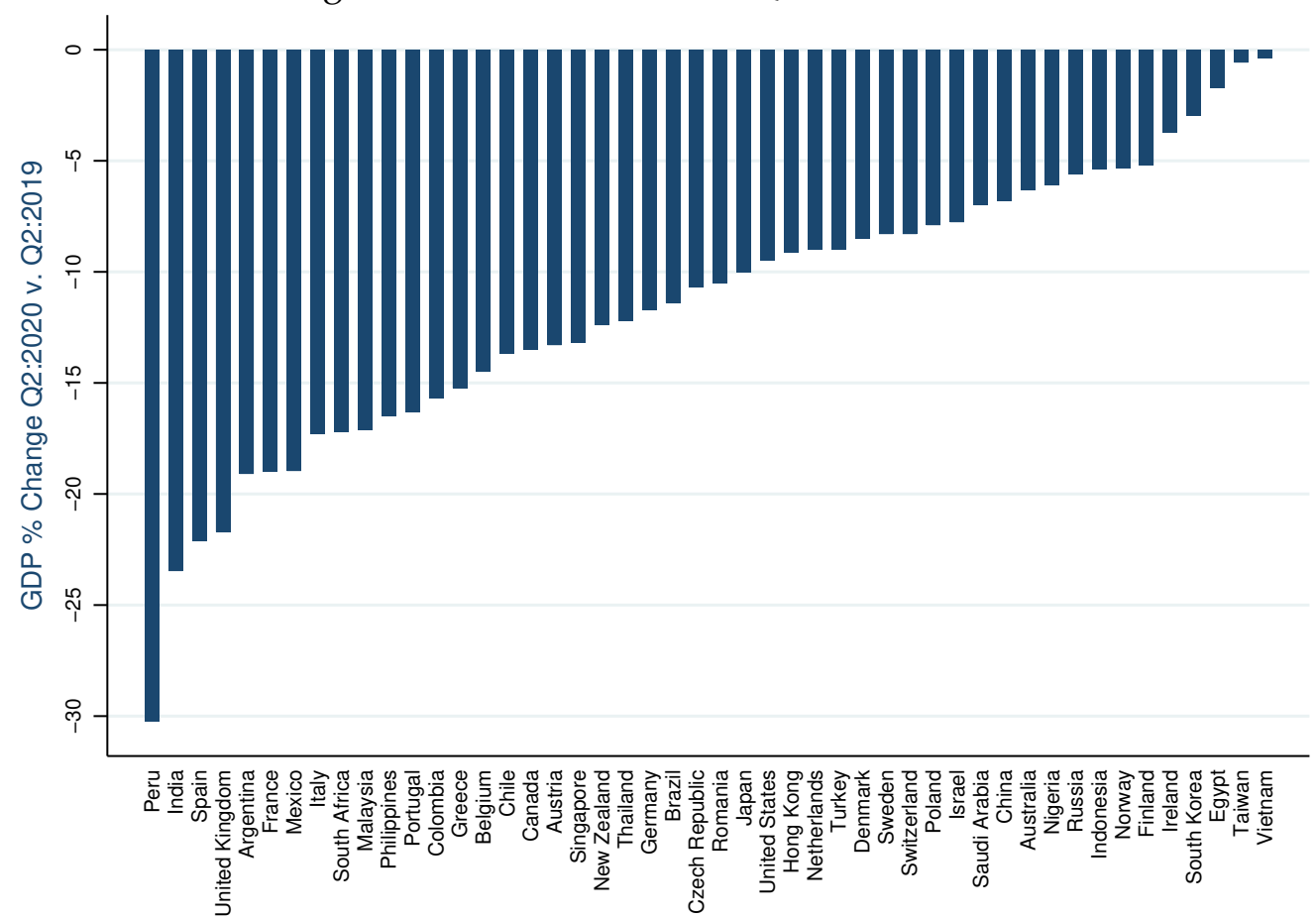

Source: http://ourworldindata.org/, https://fred.stlouisfed.org/, https://www.focus-economics.com/ and country sources.

Figure 3: Lockdown Severity and 2020:Q2 Output Declines
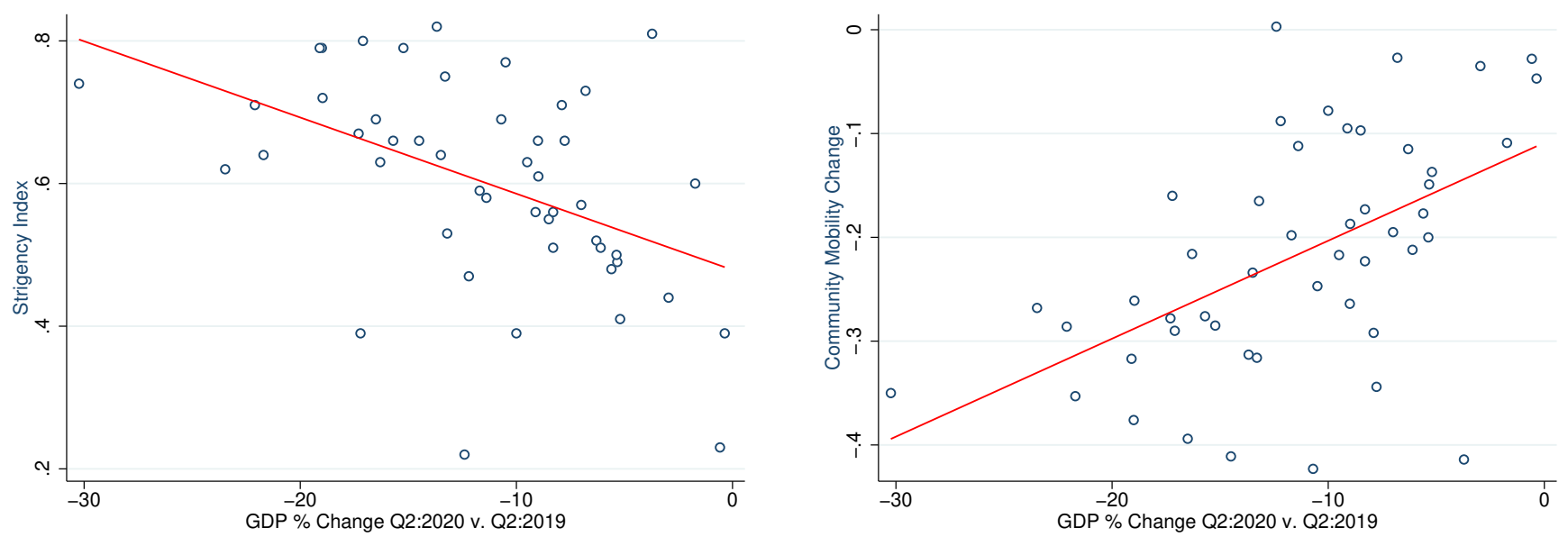

Notes: See footnotes to Figures 1 and 2 for sources of stringency index and GDP. Community mobility was retrieved from https://www.bloomberg.com/news/articles/2020-11-24/inside-bloomberg-s-covid-resilience-ranking on 11/29/2020.

\footnotetext{
${ }^{9}$ Leverage plots, influence statistics and histograms indicated outliers in Q2:2020 GDP and in several of the regressors.
} 
The results are reported in Table 3. Column (1) shows the results with the highest overall significance $\left(R_{n}^{2}=99.2\right)$ and explanatory power (robust $R_{w}^{2}=0.81$ ), and the lowest deviance coefficient (0.036). The regressors include the stringency index, non-covid excess mortality, (log of) GDP per capita, hospital beds and COVID cases. Using together a measure of lockdown severity, proxies for resource shortages and health system capacity, and COVID cases is important to avoid possible omitted variable bias (e.g. lockdown severity is likely to depend on COVID cases and deaths). Simultaneity bias is addressed by using lockdown severity and COVID variables with data up to November, 2020, which makes them less likely to be determined jointly with Q2:2020 GDP. All of the regression coefficients are significant at the 95-percent level or higher (hospital beds marginally) and have the expected signs. The regression explains roughly 81 percent of the cross-country variation in Q2:2020 GDP drops. In addition, an important result for the argument of this paper is that lockdown severity and the variables that proxy for health system resources and capacity are all significant, even after controlling for COVID infections. Non-covid excess mortality has the largest elasticity. A 100basis-points increase in this variable reduces quarterly GDP growth by 1.12 basis points, compared with 0.9 for a 100-basis points rise in the stringency index and 0.65 for a cut in GDP per capita of the same magnitude. Moreover, hospital beds have a significantly larger effect on quarterly GDP than COVID cases. Adding one bed per one-thousand people improves GDP by roughly 58 basis points, whereas an extra COVID case per one-thousand inhabitants reduces GDP growth by 35 basis points.

Columns (2)-(8) explore the robustness of the above results to potentially important modifications. Column (2) shows that adding cumulative COVID deaths is not useful. The coefficient for this variable is not significant, the rest of the coefficients change slightly, and the explanatory power, significance and deviance statistics of the regression are slightly weaker. The coefficients on stringency and beds are estimated with less precision (they are marginally significant at the 90-percent confidence level). Column (3) shows that replacing cases for November, 2020 with cumulative COVID deaths throughout end November worsens the results. The regression explains about 10 percentage points less of the cross-country variation in GDP drops and has sharply lower $R_{n}^{2}$ and higher deviance than Columns (1) and (2). Beds are no longer significant. Columns (4) and (6) show that replacing the stringency index with community mobility makes little difference. Both are statistically significant (with opposite signs because higher mobility implies a weaker lockdown), and the other coefficients are similar to those in Columns (3) and (5), respectively. Column (5) shows that removing hospital beds also weakens the results, with sharply lower $R_{n}^{2}$ and $R_{w}^{2}$ and higher deviance. Column (7) compared with Column (5) shows that using either GDP per capita or HDI yields similar results. Finally, Column (8) shows that removing hospital beds from Column (1) yields slightly weaker results. The coefficient on the stringency index rises from -0.095 to -0.128 , suggesting the possibility of omitted variable bias as lockdown severity may depend on hospital capacity.

In summary, Table 3 yields three key results: (a) non-SIR variables, particularly the proxies for differences in health system resources and capacity, are important determinants of the depth of the recessions caused by COVID-19, even after controlling for the direct effects of COVID transmission; (b) variables driving SIR dynamics also play a role, since the coefficients on COVID cases and/or deaths are statistically significant; and (c) the effects of non-SIR variables are stronger. 
Table 3: Cross-Country Regressions for Output Collapse in Q2:2020

\begin{tabular}{|c|c|c|c|c|c|c|c|c|}
\hline \multicolumn{9}{|c|}{ Dependent variable: year-on-year quarterly GDP growth as of Q2:2020 } \\
\hline Regressors & (1) & (2) & (3) & (4) & (5) & (6) & (7) & (8) \\
\hline Stringency & $\begin{array}{l}-0.095 \\
(0.048)\end{array}$ & $\begin{array}{l}-0.084 \\
(0.109)\end{array}$ & $\begin{array}{l}-0.110 \\
(0.073)\end{array}$ & & $\begin{array}{l}-0.115 \\
(0.058)\end{array}$ & & $\begin{array}{l}-0.116 \\
(0.062)\end{array}$ & $\begin{array}{l}-0.128 \\
(0.010)\end{array}$ \\
\hline Mobility & & & & $\begin{array}{c}0.186 \\
(0.025)\end{array}$ & & $\begin{array}{c}0.168 \\
(0.044)\end{array}$ & & \\
\hline $\begin{array}{c}\text { Non-covid excess } \\
\text { Mortality }\end{array}$ & $\begin{array}{l}-0.112 \\
(0.000)\end{array}$ & $\begin{array}{l}-0.108 \\
(0.000)\end{array}$ & $\begin{array}{l}-0.081 \\
(0.004)\end{array}$ & $\begin{array}{l}-0.095 \\
(0.000)\end{array}$ & $\begin{array}{l}-0.084 \\
(0.002)\end{array}$ & $\begin{array}{l}-0.090 \\
(0.001)\end{array}$ & $\begin{array}{l}-0.089 \\
(0.002)\end{array}$ & $\begin{array}{l}-0.116 \\
(0.000)\end{array}$ \\
\hline $\ln (\mathrm{GDP} p \mathrm{p})$ & $\begin{array}{c}0.065 \\
(0.000)\end{array}$ & $\begin{array}{c}0.063 \\
(0.000)\end{array}$ & $\begin{array}{c}0.047 \\
(0.001)\end{array}$ & $\begin{array}{c}0.052 \\
(0.000)\end{array}$ & $\begin{array}{c}0.050 \\
(0.000)\end{array}$ & $\begin{array}{c}0.051 \\
(0.000)\end{array}$ & & $\begin{array}{c}0.071 \\
(0.000)\end{array}$ \\
\hline Human dev. index & & & & & & & $\begin{array}{c}0.361 \\
(0.000)\end{array}$ & \\
\hline Hospital beds & $\begin{array}{c}5.801 \\
(0.053)\end{array}$ & $\begin{array}{c}5.262 \\
(0.100)\end{array}$ & $\begin{array}{c}1.649 \\
(0.644)\end{array}$ & $\begin{array}{l}2.766 \\
(0.410)\end{array}$ & & & & \\
\hline Covid cases & $\begin{array}{c}-3.51 \mathrm{E}-04 \\
(0.001)\end{array}$ & $\begin{array}{c}-3.11 \mathrm{E}-04 \\
(0.013)\end{array}$ & & & & & & $\begin{array}{c}-2.98 \mathrm{E}-04 \\
(0.005)\end{array}$ \\
\hline Covid deaths & & $\begin{array}{c}-1.49 \mathrm{E}-05 \\
(0.557)\end{array}$ & $\begin{array}{c}-6.00 \mathrm{E}-05 \\
(0.020)\end{array}$ & $\begin{array}{c}-3.85 \mathrm{E}-05 \\
(0.144)\end{array}$ & $\begin{array}{c}-6.06 \mathrm{E}-05 \\
(0.017)\end{array}$ & $\begin{array}{c}-5.15 \mathrm{E}-05 \\
(0.050)\end{array}$ & $\begin{array}{c}-5.84 \mathrm{E}-05 \\
(0.024)\end{array}$ & \\
\hline \# Observations & 35 & 35 & 35 & 35 & 35 & 35 & 35 & 35 \\
\hline$R_{w}^{2}$ & 0.812 & 0.809 & 0.711 & 0.770 & 0.713 & 0.711 & 0.702 & 0.780 \\
\hline Adjusted $R_{w}^{2}$ & 0.812 & 0.809 & 0.711 & 0.770 & 0.713 & 0.711 & 0.702 & 0.780 \\
\hline$R_{n}^{2}$ & 99.151 & 93.737 & 55.515 & 69.026 & 56.655 & 61.942 & 54.073 & 82.747 \\
\hline Deviance & 0.0363 & 0.037 & 0.0461 & 0.042 & 0.046 & 0.047 & 0.048 & 0.042 \\
\hline
\end{tabular}

Notes: All regressions were estimated using Robust MM Least Squares. The variables are deviations from their respective country means in the common sample. Numbers in parenthesis are p-values. Stringency is the Oxford stringency index divided by 100. Mobility is Google's community mobility indicator. Both stringency and mobility are 30-day averages over a period ending in late November 2020. Non-covid excess mortality is the residual of regressing the excess mortality P-Scores in Table 1 on the cumulative deaths due to COVID-19 as of end November 2020. Human dev. index is the 2019 UNDP's human development index, which combines GNI per capita, life expectancy at birth, and mean years of schooling of adults older than 25. Ln(GDP pc) is the natural log of GDP per capita in 2019. Hospital beds are per 1 million inhabitants. Covid cases are the one-month COVID cases per 1 million population for the month ending in late November 2020. Covid deaths are cumulative deaths due to Covid-19 per 1 million inhabitants through late November 2020. Q2:2020 GDP data are from the sources reported in the note to Figure 2. Data on stringency, mobility, human development index, Covid cases and Covid deaths were retrieved from https://www.bloomberg.com/news/articles/2020-11-24/inside-bloomberg-s-covid-resilience-ranking on 11/29/2020. 2019 real GDP and hospital beds are from World Bank Open Data and population data are from IMF World Economic Outlook. Hospital beds data are based on WHO figures and is for the most recent year available, which is in the 2010-2015 range for most countries.

\section{A Model of the Output-Pandemia Tradeoff}

The key feature of the model is the characterization of a pandemia as a large, transitory shock to the subsistence level of demand for health goods and services $\left(\bar{h}_{t}\right)$ in a Stone-Geary utility function that is directly related to the utilization rate $\left(m_{t}\right)$. The value of $\bar{h}_{t}$ is given by:

$$
\bar{h}_{t}=h^{\star}+z_{t} f\left(m_{t} K\right)
$$

where $h^{\star}$ is the "normal" subsistence demand for $h$ goods, $z_{t}$ is a binary variable which equals 0 in normal times and 1 when there is a pandemia, and $f(\cdot)$ is a monotonically increasing function. A pandemia lasts $j$ periods, so that $z_{t}=1$ for $t=0, \ldots, j$ and $z_{t}=0$ for $t>j$, and is a fully 
unanticipated, non-recurrent shock. ${ }^{10}$ In addition, the supply of health goods $H$ is assumed to be fixed, which is reasonable since the shock is unanticipated and key parts of the provision of health goods and services rely on forms of capital that are difficult to adjust in the short-run (e.g. hospitals, equipment, specialists, etc).

\subsection{Decentralized Competitive Equilibrium}

\subsubsection{Households}

There are two types of households, which together add up to a unit mass of agents. All agents have identical utility functions. A fraction $\gamma_{1}$ are type- 1 agents (entrepreneurs) who own all the wealth, both the capital stock used to produce non-health goods and the stock of health goods and services.

The optimization problem of an individual of type 1 is to maximize this utility function:

$$
\max _{\left\{c_{t}^{1}, l_{t}^{1}, h_{t}^{1}, d_{t+1}^{1}\right\}} \sum_{t=0}^{\infty} \beta^{t}\left(a \ln \left(c_{t}^{1}-\frac{\left(l_{t}^{1}\right)^{\omega}}{\omega}\right)+(1-a) \ln \left(h_{t}^{1}-\bar{h}_{t}\right)\right),
$$

subject to the following budget constraint,

$$
c_{t}^{1}+p_{t}^{h} h_{t}^{1}=w_{t} l_{t}^{1}-q_{t} d_{t+1}^{1}+d_{t}^{1}+\pi_{t}+p_{t}^{h} h-\tau_{t} .
$$

In the above expressions, $c_{t}^{1}$ and $h_{t}^{1}$ are consumption of non-health and health goods by an agent of type-1, respectively, and $l_{t}^{1}$ is its labor supply. In addition, $d_{t}^{1}$ and $d_{t+1}^{1}$ are the agent's holdings of existing and newly-issued public debt. Non-health goods are the numeraire, so $p_{t}^{h}$ is the relative price of health goods, $w_{t}$ is the wage rate, and $q_{t}$ is the price of government bonds, all in units of non-health goods. Type- 1 agents are the only agents who purchase public debt. They own the endowment of health goods, with an amount $h$ for each type- 1 agent, and they also collect the profits paid by firms producing non-health goods and pay lump-sum taxes, with amounts $\pi_{t}$ and $\tau_{t}$ for each type- 1 agent, respectively, both in units of non-health goods.

The utility function is time-separable, with discount factor $\beta$, and period utility is a Stone-Geary utility function of consumption of $h$ and $c$. The argument for utility of non-health consumption is of the Greenwood-Hercowitz-Huffman form (i.e. the subsistence level is determined by the disutility of labor, which removes the wealth effect on labor supply by making the marginal rate of substitution between $l_{t}^{1}$ and $c_{t}^{1}$ independent of the latter). ${ }^{11}$ At equilibrium, the parameter $a$ is the share of expenditure on non-health goods in excess of the disutility of labor relative to income net of the disutility of labor and subsistence expenditure on health goods. Similarly, $(1-a)$ is the share of excess health expenditure above its subsistence level relative to the same net income measure.

\footnotetext{
${ }^{10}$ The pandemia could also be modeled as a stochastic, non-insurable disaster shock, but modeling it as an unanticipated shock is a reasonable approximation to how COVID-19 arrived. Still, modeling it as a disaster shock is worthwhile, because it would alter precautionary saving behavior and incentivize the accumulation of buffer stocks of health goods.

${ }^{11}$ This assumption is essential for the result that aggregate allocations and the optimal lockdown are independent of agent heterogeneity, inequality and optimal transfers.
} 
Simplifying the first-order conditions of the above problem yields these optimality conditions:

$$
\begin{gathered}
\frac{1-a}{a} \frac{c_{t}^{1}-\frac{\left(l_{t}^{1}\right)^{\omega}}{\omega}}{h_{t}^{1}-\bar{h}_{t}}=p_{t}^{h} \\
\left(l_{t}^{1}\right)^{\omega-1}=w_{t} \\
\frac{c_{t+1}^{1}-\frac{\left(l_{t+1}^{1}\right)^{\omega}}{\omega}}{c_{t}^{1}-\frac{\left(l_{t}^{1}\right)^{\omega}}{\omega}}=\beta R_{t}
\end{gathered}
$$

where $R_{t} \equiv 1 / q_{t}$. Condition (4) equates type-1's marginal rate of substitution between non-health and health consumption to the corresponding relative price. Condition (5) equates the marginal disutility of labor supply to the real wage. Condition (6) equates the intertemporal marginal rate of substitution in consumption to the real return on public debt.

The second type of agents are the workers who are a fraction $\gamma_{2} \equiv 1-\gamma_{1}$ of the unit-mass of agents. The optimization problem of a single type-2 agent is given by:

$$
\max _{\left\{c_{t}^{2}, l_{t}^{2}, h_{t}^{2}\right\}} \sum_{t=0}^{\infty} \beta^{t}\left(a \ln \left(c_{t}^{2}-\frac{\left(l_{t}^{2}\right)^{\omega}}{\omega}\right)+(1-a) \ln \left(h_{t}^{2}-\bar{h}_{t}\right)\right),
$$

subject to this budget constraint,

$$
c_{t}^{2}+p_{t}^{h} h_{t}^{2}=w_{t} l_{t}^{2}+t r_{t}
$$

Here, $c_{t}^{2}$ and $h_{t}^{2}$ are consumption of non-health and health goods by an agent of type 2 , respectively, and $l_{t}^{2}$ is its labor supply. Type-2 agents collect income only from wages $\left(w_{t} l_{t}^{2}\right)$ and from government transfers in the amount $t r_{t}$ per agent.

The first-order conditions of the above problem reduce to the following optimality conditions:

$$
\begin{gathered}
\frac{1-a}{a} \frac{c_{t}^{2}-\frac{\left(l_{t}^{2}\right)^{\omega}}{\omega}}{h_{t}^{2}-\bar{h}_{t}}=p_{t}^{h}, \\
\left(l_{t}^{2}\right)^{\omega-1}=w_{t} .
\end{gathered}
$$

Condition (9) equates type-2's marginal rate of substitution between non-health and health consumption to $p_{t}^{h}$. Condition (10) equates the marginal disutility of labor supply to the real wage.

\subsubsection{Firms}

All firms are identical and the representative firm's optimization problem is:

$$
\max _{m_{t}, L_{t}} \quad \Pi_{t}=\left(m_{t} K\right)^{1-\alpha} L_{t}^{\alpha}-w_{t} L_{t}-\chi_{0} \frac{m_{t}^{\chi_{1}}}{\chi_{1}} K
$$

subject to the technlogical constraint on utilization,

$$
m_{t} \leq \bar{m}
$$


where $L_{t}$ is aggregate labor demand and $\bar{m}$ is the technologically-feasible maximum rate of utilization, which is assumed to be nonbinding. Since the capital stock is constant, utilization costs $\left(\chi_{0} \frac{m_{t}^{\chi_{1}}}{\chi_{1}} K\right)$ can be seen as the standard cost associated to faster depreciation at higher utilization or as a rental cost that increases with utilization.

The first-order conditions of the above problem yield standard marginal-productivity conditions for labor demand and the utilization rate:

$$
\begin{gathered}
(1-\alpha)\left(m_{t} K\right)^{-\alpha} L_{t}^{\alpha}=\chi_{0} m_{t}^{\chi_{1}-1}, \\
\alpha\left(m_{t} K\right)^{1-\alpha} L_{t}^{\alpha-1}=w_{t} .
\end{gathered}
$$

The marginal products of utilization and labor equal their marginal costs. For the former, the cost is determined by the firm's utilization choice and for the latter the cost is the market wage rate.

\subsubsection{Government Budget Constraint}

The government budget constraint is the following:

$$
T_{t}-T R_{t}=D_{t}-q_{t} D_{t+1}
$$

The left-hand-side is the primary balance, which equals aggregate tax revenue, $T_{t}$, minus total transfer payments, $T R_{t}$. The right-hand-side equals the repayment of existing debt net of the resources raised by selling new debt.

The fiscal structure could be simplified by abstracting from public debt so that transfers to type2 agents are paid by lump-sum taxes paid by type- 1 agents and the government's budget is balanced each period. Debt is introduced just so that we can highlight some implications of debtfinanced transfers for fiscal solvency, but the two formulations are equivalent because the taxes are non-distortionary (i.e. debt is Ricardian). For a given policy of transfers funded with lump-sum taxes, the debt-equivalent formulation (without taxes) is given by the sequence of debt issuance $q_{t} D_{t+1}=D_{t}+T R_{t}$, starting from a given initial debt $D_{0}$. The debt formulation requires, however, that the intertemporal government budget constraint must hold, so the present discounted value of the primary balance as of any date $t$ must match the outstanding debt as of that date. Hence, if it is optimal to increase transfers during a pandemia (as we show later) and the transfers are debtfinanced, the debt accumulated during the $j$ periods of the pandemia $\left(D_{j+1}\right)$ is sustainable only if the stream of primary balances for $t>j$ increases so that their present value equals $D_{j+1}$, which can be accomplished by imposing lump-sum taxes on type-1 agents.

\subsubsection{Competitive Equilibrium with and without Pandemia}

The decentralized competitive equilibrium (DCE) is defined by sequences of individual allocations $\left\{c_{t}^{1}, c_{t}^{2}, h_{t}^{1}, h_{t}^{2}, l_{t}^{1}, l_{t}^{2}, d_{t+1}^{1}\right\}_{t=0}^{\infty}$, aggregate allocations $\left\{m_{t}, L_{t}, C_{t}, \Pi_{t}\right\}_{t=0}^{\infty}$, and prices $\left\{R_{t}, p_{t}^{h}, w_{t}\right\}_{t=0}^{\infty}$ such that: (a) the optimality conditions of type- 1 and type-2 agents hold, (b) the optimality conditions 
of the representative firm hold, (c) the following market-clearing conditions:

$$
\begin{gathered}
\gamma_{1} l_{t}^{1}+\gamma_{2} l_{t}^{2}=L_{t}, \\
\gamma_{1} h_{t}^{1}+\gamma_{2} h_{t}^{2}=H
\end{gathered}
$$

and (d) the following aggregation conditions hold:

$$
\begin{gathered}
\gamma_{1} d_{t+1}^{1}=D_{t+1}, \\
\gamma_{1} \tau_{t}=T_{t} \\
\gamma_{2} t r_{t}=T R_{t}, \\
\gamma_{1} h=H, \\
\gamma_{1} \pi_{t}=\Pi_{t} \\
\gamma_{1} c_{t}^{1}+\gamma_{2} c_{t}^{2}=C_{t} .
\end{gathered}
$$

The budget constraints of the agents, the definition of profits and the above market-clearing and aggregation conditions yield the following resource constraint:

$$
C_{t}=\left(m_{t} K\right)^{1-\alpha} L_{t}^{\alpha}-\chi_{0} \frac{m_{t}^{\chi_{1}}}{\chi_{1}} K
$$

Since the only shock to the economy is the unanticipated, temporary hike in $\bar{h}_{t}$ during the pandemia, and since there are no endogenous mechanisms to induce dynamics, the DCE separates into pandemia (P) and no-pandemia (NP) phases, and within each prices and allocations are constant. The DCE has a closed-form solution. To characterize the DCE solution, consider first that, since preferences are identical, labor is homogeneous, and all agents are paid the same wage, conditions (5) and (10) imply that all agents offer the same labor supply, which must equal labor demand at equilibrium: $l_{t}^{1}=l_{t}^{2}=L_{t}$. Therefore, using the labor demand and supply conditions, considering that both must be equal at the equilibrium wage, yields this expression:

$$
L_{t}^{\omega-1}=\alpha\left(m_{t} K\right)^{1-\alpha} L_{t}^{\alpha-1}
$$

This condition together with the firm's optimality condition for utilization yields the following expression for the labor allocation as a function of the utilization rate:

$$
L_{t}=\left(\frac{\chi_{0} \alpha K}{1-\alpha}\right)^{\frac{1}{\omega}} m_{t}^{\frac{\chi_{1}}{\omega}} .
$$

Using the above result, factor allocations can be solved for using conditions (13) and (14):

$$
m_{t}=m^{\star}=\left(\chi_{0}^{\alpha-\omega} \alpha^{\alpha}(1-\alpha)^{\omega-\alpha} K^{\alpha(1-\omega)}\right)^{\frac{1}{\chi_{1} \omega+\alpha \omega-\omega-\chi_{1} \alpha}}
$$




$$
L_{t}=l_{t}^{1}=l_{t}^{2}=L^{\star}=\left(\chi_{0}^{\alpha-1} \alpha^{\chi_{1}+\alpha-1}(1-\alpha)^{1-\alpha} K^{(1-\alpha)\left(\chi_{1}-1\right)}\right)^{\frac{1}{\chi_{1} \omega+\alpha \omega-\omega-\chi_{1} \alpha}} .
$$

Given the above, it is straightforward to obtain equilibrium solutions for output, profits, wages and aggregate consumption using other optimality conditions and the resource constraint:

$$
\begin{gathered}
Y_{t}=Y^{\star}=\left(m^{\star} K\right)^{1-\alpha} L^{\star \alpha} \\
\Pi_{t}=\gamma_{1} \pi^{\star}=(1-\alpha)\left(1-\frac{1}{\chi_{1}}\right) Y^{\star}>0 \\
w_{t}=w^{\star}=\left(L^{\star}\right)^{\omega-1} \\
C_{t}=C^{\star}=Y^{\star}-\chi_{0} \frac{\left(m^{\star}\right)^{\chi_{1}}}{\chi_{1}} K .
\end{gathered}
$$

Note two important properties of the aggregate DCE allocations, profits and wages solved above: First, they are independent of heterogeneity and inequality in wealth, income and consumption, as is evident by the fact that $\gamma_{1}$ and $\gamma_{2}$ do not enter in the solutions. Second, they are the same during the $\mathrm{P}$ and NP phases (i.e. for $t=0, \ldots, j$ and for $t>j$ ).

In contrast with the aggregate allocations, individual consumption allocations of health and nonhealth goods and the relative price of those goods differ in the P and NP phases in the DCE. The equilibrium prices are:

$$
\begin{aligned}
p_{t}^{\star h P} & =\frac{1-a}{a} \frac{C^{\star}-\frac{\left(L^{\star}\right)^{\omega}}{\omega}}{H-h^{\star}-f\left(m^{\star} K\right)} \text { for } t=0, \ldots, j, \\
p_{t}^{\star h N P} & =\frac{1-a}{a} \frac{C^{\star}-\frac{\left(L^{\star}\right)^{\omega}}{\omega}}{H-h^{\star}} \text { for } t>j .
\end{aligned}
$$

Prices are higher during the pandemia because of the direct effect on demand for health goods and services due to higher $\bar{h}_{t}$. In turn, this rise in $p_{t}^{\star h P}$ worsens income inequality because it increases the value of the endowment of health goods owned by type- 1 agents.

The solutions of the consumption allocations across agents are straightforward applications of the linear expenditure system implied by the Stone-Geary preferences. In particular, using conditions (4) and (9) together with the budget constraints of the two types of agents and the above results for aggregate variables we can obtain solutions for the individual consumption allocations as functions of relative prices and the subsistence demand for health:

$$
\begin{gathered}
c_{t}^{\star 1}\left(p_{t}^{\star h}, \bar{h}_{t}\right)=a\left[\pi^{\star}+p_{t}^{\star h} h-\tau_{t}+\left(L^{\star}\right)^{\omega}-p_{t}^{\star h} \bar{h}_{t}-\frac{\left(L^{\star}\right)^{\omega}}{\omega}\right]+\frac{\left(L^{\star}\right)^{\omega}}{\omega} \\
c_{t}^{\star 2}\left(p_{t}^{\star h}, \bar{h}_{t}\right)=a\left[t r_{t}+\left(L^{\star}\right)^{\omega}-p_{t}^{\star h} \bar{h}_{t}-\frac{\left(L^{\star}\right)^{\omega}}{\omega}\right]+\frac{\left(L^{\star}\right)^{\omega}}{\omega} \\
h_{t}^{\star 1}\left(p_{t}^{\star h}, \bar{h}_{t}\right)=\frac{1-a}{p_{t}^{\star h}}\left[\pi^{\star}+p_{t}^{\star h} h-\tau_{t}+\left(L^{\star}\right)^{\omega}-p_{t}^{\star h} \bar{h}_{t}-\frac{\left(L^{\star}\right)^{\omega}}{\omega}\right]+\bar{h}_{t}
\end{gathered}
$$




$$
h_{t}^{\star 2}\left(p_{t}^{\star h}, \bar{h}_{t}\right)=\frac{1-a}{p_{t}^{\star h}}\left[t r_{t}+\left(L^{\star}\right)^{\omega}-p_{t}^{\star h} \bar{h}_{t}-\frac{\left(L^{\star}\right)^{\omega}}{\omega}\right]+\bar{h}_{t}
$$

Expressing individual consumption allocations as functions of $\left(p_{t}^{\star h}, \bar{h}_{t}\right)$ is useful because these are the only two variables that cause the allocations to differ in the P and NP phases. Both $\bar{h}_{t}$ and $p_{t}^{\star h}$ are higher in the $\mathrm{P}$ phase, affecting individual consumption allocations as explained below.

Assume $t r_{t}=0$ (i.e. a DCE without transfers either in normal times or during the pandemia), or alternatively, assume that transfers are unchanged when the pandemia hits. It follows from (36) that $c^{\star 2 P}\left(p^{\star h P}, h^{\star}+f\left(m^{\star} K\right)\right)<c^{\star 2 N P}\left(p^{\star h N P}, h^{\star}\right)$, because $p_{t}^{\star h} \bar{h}_{t}$ rises during the pandemia and the rest of the variables that determine non-health consumption of type-2 agents are unaffected by the pandemia. The intuition is that type-2 agents need to redirect some of their income to pay for the subsistence level of health, which increased both in quantity and in price. Since aggregate consumption $C^{\star}$ is unchanged, it must be that $c^{\star 1 P}\left(p^{\star h P}, h^{\star}+f\left(m^{\star} K\right)\right)>c^{\star 1 N P}\left(p^{\star h N P}, h^{\star}\right)$. For these agents, the rise in the value of the endowment of health goods exceeds the increase in the cost of the subsistence level of health. Hence, during a pandemia, non-health consumption of type-1 (type-2) agents rises (falls). The same applies to excess non-health consumption relative to the disutility of labor. It rises for type- 1 agents and falls for type-2 agents.

The responses of health consumption differ from those of non-health consumption. In particular, $h_{t}^{2}$ rises but $h_{t}^{1}$ falls. The direct effect of higher $\bar{h}_{t}$ on demand for health goods is the same for both agents, but the income effect of higher $p_{t}^{h}$ reducing the real value of income is stronger for entrepreneurs as the value of profits from the non-health sector in units of health goods falls. Excess health consumption (i.e., net of $\bar{h}_{t}$ ) falls for both agents, however, because even for type- 2 agents the adverse income effects of higher prices imply that the increase in $\bar{h}_{t}$ exceeds that in $h_{t}^{2}$. Overall, type2 agents suffer more with the pandemia, because they always consume less of all goods than type- 1 agents and the pandemia causes their excess consumption of both health and nonhealth goods to fall, while for type-1 agents excess consumption of non-health goods rises.

The need to keep consumption of all goods above their subsistence levels imposes an upper bound on the set of $p_{t}^{\star h P}$ that can be supported as a DCE. In particular, the results in (36) and (38) imply that, in order for type-2 agents to keep $h_{t}^{\star 2}$ and $c_{t}^{\star 2}$ above $\bar{h}_{t}$ and $L_{t}^{\omega} / \omega$, respectively, their residual income must satisfy: $t r_{t}+\left(L^{\star}\right)^{\omega}-p_{t}^{\star h} \bar{h}_{t}-\frac{\left(L^{\star}\right)^{\omega}}{\omega}>0$. Solving for $p_{t}^{\star h P}$ yields:

$$
p_{t}^{\star h P}<\hat{p}^{\star h P} \equiv \frac{t r^{P}+\left(L^{\star}\right)^{\omega}\left(\frac{\omega-1}{\omega}\right)}{h^{*}+f\left(m^{\star} K\right)}
$$

where $\operatorname{tr}^{P}$ is a given value of exogenous transfers provided during the pandemia. Hence, the jump in $p_{t}^{\star h P}$ caused by $f\left(m^{\star} K\right)$ during a pandemia cannot reach $\hat{p}^{\star h P}$, because otherwise type-2 agents hit their subsistence consumption levels triggering the Inada conditions of their preferences. The market price, which depends on the aggregate demand for health goods, would still be well-defined by condition (33), but it cannot be an equilibrium because type-2 agents saturate the health system.

Combining the above result with the pricing condition (33) implies that $f(\cdot)$ cannot exceed this 
upper bound:

$$
f\left(m^{\star} K\right)<\frac{H}{1+\frac{1-a}{a} \frac{c^{\star}-\left(L^{\star \omega}\right) / \omega}{\operatorname{tr}^{P}+(\omega-1)\left(L^{\star \omega}\right) / \omega}}-h^{*},
$$

where $c^{\star}, L^{\star}, m^{\star}$ are the DCE allocations independent of $f(\cdot)$ (see eqns. (27), (28) and (32)).

If debt is used to pay for transfers, the real interest rate is solved for by plugging the solutions obtained above in the Euler equation of type- 1 agents (eq. (6)). Since $L^{\star}$ is constant at all times, and since consumption of type- 1 agents shifts from a higher level in the $\mathrm{P}$ phase to a lower level in the NP phase, the interest rate equals $1 / \beta$ in all periods except between $t+j$ and $t+j+1$ (the transition from pandemia to non-pandemia). The interest rate on debt sold that period is:

$$
R_{t+j}=\frac{c_{t+j+1}^{\star 1 N P}-\frac{\left(L^{\star}\right)^{\omega}}{\omega}}{\beta\left(c_{t+j}^{\star 1 P}-\frac{\left(L^{\star}\right)^{\omega}}{\omega}\right)} .
$$

Hence, given that $c^{\star 1 P}>c^{\star 1 N P}$, the interest rate falls in the last period of the pandemia.

Finally, to characterize the effects of the pandemia on consumption inequality, it is useful to focus on the ratio of excess consumption of type- 1 to type- 2 agents denoted $\Omega_{t}^{\star}$. Dividing eq. (35) by (36), or (37) by (38), yields:

$$
\Omega_{t}^{\star}=\frac{\pi^{\star}+p_{t}^{\star h} h-\tau_{t}+\left(L^{\star}\right)^{\omega}-p_{t}^{\star} h \bar{h}_{t}-\frac{\left(L^{\star}\right)^{\omega}}{\omega}}{t r_{t}+\left(L^{\star}\right)^{\omega}-p_{t}^{\star h} \bar{h}_{t}-\frac{\left(L^{\star}\right)^{\omega}}{\omega}}
$$

Across the two types of agents in the DCE, this ratio is the same for non-health consumption or for health consumption, and the ratio itself satisfies $\Omega_{t}^{\star}>1$. This is clearly true for the DCE without transfers, and when transfers are present it holds because we assume that $\tau_{t}<\pi^{\star}+p_{t}^{\star h} h$ (i.e. per-capita transfers never exceed the non-wage income of type- 1 agents). Moreover, the ratio is constant at different levels with and without pandemia, and satisfies $\Omega^{\star P}>\Omega^{\star N P}$ so that consumption inequality worsens temporarily with a pandemia.

Since both agents supply the same labor, collect the same wages, and have the same $\bar{h}$, the movements in $\Omega_{t}^{\star}$ also capture the changes in income inequality due to the pandemia. Type- 1 agents own the firms and the endowment of $H$, so their income includes, in addition to wages, the profits from non-health goods production and the sales of health goods. The adverse effect of the pandemia on income inequality occurs because the hike in the relative price of health goods induces regressive income redistribution as the income from sales of those goods that type-1 agents collect rises.

\subsection{Social Planner's Problem}

The social planner solves the following optimization problem:

$$
\begin{aligned}
& \max _{\left\{c_{t}^{j}, l_{t}^{j}, h_{t}^{j}, m_{t}\right\}} \phi\left\{\gamma_{1} \sum_{t=0}^{\infty} \beta^{t}[\right.\left.\left.a \ln \left(c_{t}^{1}-\frac{\left(l_{t}^{1}\right)^{\omega}}{\omega}\right)+(1-a) \ln \left(h_{t}^{1}-\bar{h}_{t}\right)\right]\right\} \\
&+(1-\phi)\left\{\gamma_{2} \sum_{t=0}^{\infty} \beta^{t}\left[a \ln \left(c_{t}^{2}-\frac{\left(l_{t}^{2}\right)^{\omega}}{\omega}\right)+(1-a) \ln \left(h_{t}^{2}-\bar{h}_{t}\right)\right]\right\}
\end{aligned}
$$


subject to resource constraints on labor, health goods, and non-health goods, the technological constraint on utilization, and the subsistence demand for health:

$$
\begin{gathered}
\gamma_{1} l_{t}^{1}+\gamma_{2} l_{t}^{2}=L_{t}, \\
\gamma_{1} h_{t}^{1}+\gamma_{2} h_{t}^{2}=H, \\
\gamma_{1} c_{t}^{1}+\gamma_{2} c_{t}^{2} \equiv C_{t}=\left(m_{t} K\right)^{1-\alpha} L_{t}^{\alpha}-\chi_{0} \frac{m_{t}^{\chi_{1}}}{\chi_{1}} K, \\
m_{t} \leq \bar{m}, \\
\bar{h}_{t}=h^{\star}+z_{t} f\left(m_{t} K\right) .
\end{gathered}
$$

The social welfare function is standard, with weight $\phi(1-\phi)$ on type- 1 (type-2) agents, and the ratio of these weights is denoted $\Omega^{s p} \equiv \phi /(1-\phi)$. As in the DCE, $\bar{m}$ is assumed to be nonbinding.

\subsubsection{Socially Optimal Allocations}

The social planner's equilibrium (SPE) can be characterized as the set of allocations that satisfy the constraints of the planner's problem and the following optimality conditions:

$$
\begin{gathered}
l_{t}^{1}=l_{t}^{2}=L_{t}=\left(\alpha\left(m_{t} K\right)^{1-\alpha}\right)^{\frac{1}{\omega-\alpha}} \\
(1-\alpha)\left(\frac{L_{t}}{m_{t} K}\right)^{\alpha}=\chi_{0} m_{t}^{\chi_{1}-1}+\frac{1-a}{a} \frac{\left(C_{t}-\frac{L_{t}^{\omega}}{\omega}\right)}{H-\bar{h}_{t}} z_{t} f^{\prime}\left(m_{t} K\right) \\
\frac{h_{t}^{1}-\bar{h}_{t}}{h_{t}^{2}-\bar{h}_{t}}=\Omega^{s p} \\
\frac{c_{t}^{1}-\frac{\left(l_{t}^{1}\right)^{\omega}}{\omega}}{c_{t}^{2}-\frac{\left(l_{t}^{2}\right)^{\omega}}{\omega}}=\Omega^{s p}
\end{gathered}
$$

The planner sets allocations at two different constant levels for the P and NP phases. As we show below, aggregate allocations are lower in the P phase. The conditions in (44) show that the planner aligns with the DCE in that it allocates the same labor supply to both agents, and the total labor allocation equates the marginal disutility of labor with the marginal product of labor.

Conditions (45)-(47) are essential to this paper's argument. Condition (45) determines the planner's optimal utilization choice and it drives the planner's incentive to lockdown the economy. It differs from its counterpart-equation (13) in the DCE-in that, during a pandemia, the social marginal cost of utilization in the right-hand-side of (45) exceeds its private counterpart by the amount $p_{t}^{h, s p} f^{\prime}\left(m_{t} K\right)$ where $p_{t}^{h, s p} \equiv \frac{1-a}{a} \frac{\left(C_{t}-\frac{L_{t}^{\omega}}{\omega}\right)}{H-h_{t}}$ is the social price of health goods. Hence, utilization is inefficiently chosen in the DCE during a pandemia, because firms do not internalize the marginal social cost of utilization. This cost exceeds the private one because of the marginal social value of lowering utilization to relax the degree of saturation of the health system by hampering the increase in $\bar{h}_{t}$ due to the pandemia. As a result, the planner reduces utilization and this reduces 
labor demand, output, profits and wages, giving rise to the output-pandemia tradeoff.

The SPE does not have a closed-form solution because of the non-linear nature of condition (45). Using this condition together with (44), the optimal utilization rate (i.e. the optimal lockdown) can be represented as the solution to the following non-linear equation in $m_{t}$ :

$$
\begin{aligned}
& (1-\alpha)\left(\frac{\left(\alpha\left(m_{t} K\right)^{1-\alpha}\right)^{\frac{1}{\omega-\alpha}}}{m_{t} K}\right)^{\alpha}-\chi_{0} m_{t}^{\chi_{1}-1}= \\
& \quad \frac{1-a}{a}\left[\frac{\left.\left(m_{t} K\right)^{1-\alpha}\left(\left(\alpha\left(m_{t} K\right)^{1-\alpha}\right)^{\frac{\alpha}{\omega-\alpha}}\right)-\chi_{0} \frac{m_{t}^{\chi_{1}}}{\chi_{1}} K-\frac{\left(\left(\alpha\left(m_{t} K\right)^{1-\alpha}\right)^{\frac{\omega}{\omega-\alpha}}\right)}{\omega}\right]}{H-\bar{h}_{t}}\right] z_{t} f^{\prime}\left(m_{t} K\right) .
\end{aligned}
$$

Without pandemia, $z_{t}=0$ and this equation collapses to the closed-form solution for utilization in the DCE, because there is no externality affecting the choice of $m_{t}$. Labor, output, and aggregate consumption are therefore the same as well. During the pandemia, utilization is lower because of its higher marginal social cost, but notice that it retains the property of the DCE that it is independent of individual allocations and now also of the planner's welfare weights. As a result, the planner's aggregate allocations for labor and production in the pandemia phase also retain this property.

The above results imply that in this model the utilization externality and the optimal lockdown do not interact with the planner's incentives to redistribute (i.e., with inequality and agent heterogeneity). The planner's utilization choice depends on $f^{\prime}\left(m_{t} K\right)$ and $p_{t}^{h, s p}$, which are determined by aggregate variables. The planner determines first aggregate utilization and non-health GDP and then allocates health and non-health GDP to keep the ratios of excess consumption across agents equal to each other and equal to $\Omega^{s p}{ }^{12}$ This also implies that the planner's aggregate allocations and the utilization externality are identical in a representative-agent version of the model (i.e., for $\left.\gamma_{1}=1\right)$.

Conditions (46) and (47) are important because they drive the planner's incentives to redistribute resources across agents during the pandemia. The planner sets the (inverse) ratios of marginal utilities of health and non-health consumption across agents equal to the ratio its welfare weights. The extent to which redistribution is relevant depends on the extent to which $\Omega^{s p}$ differs from $\Omega^{\star P}$ and $\Omega^{\star N P}$ (recall that in the DCE we showed that $\Omega^{\star P}>\Omega^{\star N P}>1$ ).

Consider three scenarios. First, a case with $\Omega^{s p}=1$ (i.e. $\phi=1 / 2$ ). This corresponds to a utilitarian social welfare function in which the planner weighs each agent equally. ${ }^{13}$ The planner redistributes resources so as to equalize consumption of health and non-health goods across agents. Second, a case with $\Omega^{s p}=\Omega^{\star N P}$ (i.e. $\phi=\Omega^{\star N P} /\left(1+\Omega^{\star N P}\right)$ ). This is an application of the First Welfare Theorem in which the DCE without pandemia is supported as an SPE. ${ }^{14}$ The planner has no

\footnotetext{
${ }^{12}$ The resource constraints and conditions (46) and (47) imply also that $p_{t}^{h, s p}=\left(c_{t}^{i}-\frac{\left.\left(l_{t}^{i}\right)^{\omega}\right)}{\omega} /\left(h_{t}^{i}-\bar{h}_{t}\right)\right.$ for $i=1,2$.

${ }^{13}$ In this case, $\phi$ can be ignored because it becomes a common factor for the utility of both agent types in the social welfare function, and the planner's allocations become independent of $\phi$.

${ }^{14}$ This is evident because with $\Omega^{s p}=\Omega^{\star N P}$ and $z_{t}=0$ for all $t$ the SPE's optimality conditions are identical to those of the DCE without pandemia.
} 
incentive to redistribute without a pandemia, but will still want to redistribute during a pandemia because $\Omega^{s p}=\Omega^{\star N P}<\Omega^{\star P}$. Third, a case with $\Omega^{s p}>\Omega^{\star N P}$ (i.e. $\left.\Omega^{\star N P} /\left(1+\Omega^{\star N P}\right)<\phi \leq 1\right)$. This is a case with bias in favor of entrepreneurs, because the planner weighs type- 1 agents by more than what the inequality implicit in $\Omega^{\star N P}$ indicates. We will show later that when this is the case it is possible for the optimal policies to be Pareto efficient (i.e. the lifetime utility of both agents increases relative to the DCE). In light of these results, the analysis that follows focuses on $\Omega^{s p} \in[1, \infty$ ) (or $\phi \in[1 / 2,1])$.

It is worth noting that if $\phi<\Omega^{\star N P} /\left(1+\Omega^{\star N P}\right)$, the planner will engage in redistribution in favor of type- 2 agents relative to the DCE even without pandemia. Still, the optimal transfers solely due to the pandemia can be separated from the those that are optimal in "normal times" so as to focus on the additional redistribution that is socially desirable when a pandemia hits.

Given the above intuition for the utilization externality and the distributional incentives of the planner, we can now characterize the solution of the planner's problem when the pandemia is present. The solution to the non-linear equation (48) yields the planner's optimal utilization rate $m_{t}^{s p}$, and once it is known it can be used to determine the rest of the SPE allocations: $L_{t}^{s p}, C_{t}^{s p}, c_{t}^{1 s p}, c_{t}^{2 s p}$, $h_{t}^{1 s p}$, and $h_{t}^{2 s p}$. It is evident that there are no distributional incentives affecting the utilization choice because $\phi, \gamma_{1}$ and $\gamma_{2}$ do not enter in eq.(48). The higher social marginal cost of utilization leads the planner to reduce $m_{t}^{s p}$. Condition (44) then implies that aggregate and individual labor allocations fall, and since both labor and utilization fall, output and $C_{t}$ also fall. This is again the outputpandemia tradeoff: The planner internalizes that by reducing utilization it weakens the pandemia, but it also takes into account that lowering utilization has output and consumption costs.

The drops in utilization, output and consumption chosen by the planner trigger distributional incentives, because as $C_{t}^{s p}$ falls, the planner wants to keep consumption ratios aligned with $\Omega^{s p}$. Given the SPE's aggregate allocations, the planner assigns to type-2 agents these consumption allocations:

$$
\begin{gathered}
c_{t}^{2 s p}=\frac{C_{t}^{s p}-\frac{\left(L_{t}^{s p}\right)^{\omega}}{1+\gamma_{1}\left(\Omega^{s p}-1\right)}+\frac{\left(L_{t}^{s p}\right)^{\omega}}{\omega},}{h_{t}^{2 s p}=\frac{H-h^{*}-z_{t} f\left(m_{t}^{s p} K\right)}{1+\gamma_{1}\left(\Omega^{s p}-1\right)}+h^{*}+z_{t} f\left(m_{t}^{s p} K\right) .}
\end{gathered}
$$

The denominators of the first terms in the right-hand-side of the above expressions are equal to 1 for the utilitarian planner ( since $\Omega^{s p}=1$ ), and the solutions give the consumption levels that are common for all agents. For $\Omega^{s p}>1$, these expressions yield consumption levels for type-2 agents that are lower than for type-1 agents. Type-2 (type-1) agents receive "below average" ("above average") consumption levels so that market-clearing in health and non-health goods holds. As explained earlier, the size of $\Omega^{s p}$ determines the degree of consumption inequality that is optimal for the planner. For $\Omega^{s p}=\Omega^{\star N P}\left(\right.$ recall $\left.\Omega^{\star N P}>1\right)$, this yields the same consumption allocations and the same inequality as in the DCE so that no redistribution is optimal without a pandemia. 


\subsubsection{Decentralization \& Optimal Policies}

The social planner's allocations can be implemented as a competitive equilibrium by imposing a lockdown (i.e. a binding limit on utilization) and providing transfers to type-2 agents. The optimal design of these two policies is characterized below.

Optimal Lockdown: The planner's optimal utilization rate can be decentralized using various instruments to correct the utilization externality. Since COVID-19 arrived as a large, unexpected shock that required an urgent response to the threat of saturation of health systems, it is reasonable to consider a lockdown as the policy instrument, instead of standard policy instruments (e.g. taxes) that would have been too slow and cumbersome to implement. The optimal lockdown is obtained by implementing the following policy rule:

$$
\begin{aligned}
& m_{t} \leq m_{t}^{s p} \quad \text { for } t=0, \ldots, j \\
& m_{t} \leq \bar{m} \quad \text { for } t>j .
\end{aligned}
$$

Since the utilization externality increases the marginal cost of utilization relative to the DCE and $\bar{m}$ is not binding in the DCE, it must be the case that $m_{t}^{s p}<m^{\star}<\bar{m}$ for $t=0, \ldots, j$. Recall also that in the DCE, $m^{\star}$ is the optimal utilization rate with or without pandemia and that, since there is no utilization externality without pandemia, $m_{t}^{s p}=m^{\star}$ for $t>j$.

Optimal Transfers: By imposing the planner's health and non-health consumption allocations for type-2 agents (eqns. (49) and (50)) on these agents' budget constraint in the DCE solution (eq. (8)), it follows that the optimal policy rules for government transfers during and post the pandemia are

$$
\begin{array}{r}
T R_{t}^{s p, P}=\gamma_{2}\left[\left\{\frac{C_{t}^{s p}-\frac{\left(L_{t}^{s p}\right)^{\omega}}{\omega}}{1+\gamma_{1}\left(\Omega^{s p}-1\right)}+\frac{\left(L_{t}^{s p}\right)^{\omega}}{\omega}+p_{t}^{h, s p}\left(\frac{H-h^{*}-f\left(m_{t}^{s p} K\right)}{1+\gamma_{1}\left(\Omega^{s p}-1\right)}+h^{*}+f\left(m_{t}^{s p} K\right)\right)\right\}\right. \\
T R_{t}^{s p, N P}=\gamma_{2}\left[\left\{\frac{C^{\star}-\frac{\left(L^{\star}\right)^{\omega}}{\omega}}{1+\gamma_{1}\left(\Omega^{s p}-1\right)}+\frac{\left(L^{\star}\right)^{\omega}}{\omega}+p^{\star h}\left(\frac{H-h^{*}}{1+\gamma_{1}\left(\Omega^{s p}-1\right)}+h^{*}\right)\right\}-\left(L^{\star}\right)^{\omega}\right] \quad \text { for } t>j . \\
\end{array}
$$

In the expressions inside square brackets, the terms in braces represent the total value of nonhealth and health consumption of type-2 agents, and the term $\left(L_{t}^{s p}\right)^{\omega}$ is these agents' wage income. Hence, the optimal transfer finances the gap between the planner's desired allocation of total consumption to type- 2 agents and the wages they collect (all in units of nonhealth goods). The optimal transfers are constant at different levels in the P and NP phases, just like the SPE's allocations.

The planner takes into account that a pandemia always worsens income inequality (even with an optimal lockdown), as it increases the market income of type-1 agents relative to that of type- 2 agents, since the latter only earn wages while the former collect profits and sales of $H$ in addition 
to wages. The planner internalizes that the relative price of health goods rises, making health-good purchases costlier and income from selling health goods larger, and that without policy intervention the overall result of these effects would move type-2 agents closer to their subsistence levels of health and non-health goods. To correct for this, the planner intervenes to redistribute income and consumption from type- 1 to type- 2 agents by more than it does in normal times without pandemia. If $\Omega^{s p}=\Omega^{\star N P}$, there is no redistribution in normal times $\left(T R^{s p, N P}=0\right)$, but the planner still redistributes during the pandemia. Hence, the planner has incentives to intervene in the DCE so as to both reduce utilization (to tackle the utilization externality) and redistribute resources across agents (to redistribute the decline in aggregate output across agents and maintain their ratio of excess consumptions equal to $\Omega^{s p}$ ).

The planner chooses $m_{t}^{s p}$ independently of inequality but it is critical to note that the lockdown itself contributes to mitigate the effects of the pandemia on inequality. This is because the lockdown reduces the spike in the price of health goods that drives the regressive effect on income and thereby mitigates the increase in consumption inequality too. As a result, the lower $p_{t}^{h, s p}$ that results from the lockdown reduces the size of the transfers that the planner needs to provide during the pandemia, as equation (53) shows.

As explained earlier, the planner can pay for the optimal transfers during the pandemia with lump-sum taxes on type- 1 agents maintaining a balanced budget, or it can finance them by selling debt to those agents. Using debt, the equilibrium interest rates would be given by $R_{t}=1 / \beta$ for $t=$ $0, . ., j-1$ or $t>j$ and $R_{j}=\left(c_{j+1}^{1 s p}-\frac{\left(L_{j+1}^{s p}\right)^{\omega}}{\omega}\right) /\left[\beta\left(c_{j}^{1 s p}-\frac{\left(L_{j}^{s p}\right)^{\omega}}{\omega}\right)\right]$. Since transfers are constant during the pandemia and the interest rate differs from $1 / \beta$ only in period $j$, the planner would arrive at the end of the pandemia with a debt stock $D_{j+1}^{s p}=\left(1 / R_{j}\right)\left[T R^{s p, P} \sum_{i=0}^{j-1} \beta^{i}+\beta^{j-1} D_{0}\right]$. In order to maintain fiscal solvency after the pandemia (i.e. satisfy the intertemporal government budget constraint), the government can impose lump-sum taxes $T_{t}$ for $t>j$ such that the present discounted value of tax revenue equals $D_{j+1}^{s p}$. The specific sequence of these taxes is undetermined. Any sequence that satisfies the solvency condition yields the same outcome because the taxes are non-distortionary. For instance, since $R_{t}=1 / \beta$ for $t>j$, a constant lump-sum tax $\bar{T}=(1-\beta) D_{j+1}^{s p}$ satisfies the solvency condition. A tax paying all the debt in one period $\left(T_{j+1}=D_{j+1}^{s p}\right)$ is also consistent with solvency, but is akin to a default in which the government "pays" all the debt at $t=j+1$ by simply taxing away the entire debt repayment. The planner has no reason to prefer either debt or taxes to pay for transfers during the pandemia, or any particular sequence of taxes post-pandemia consistent with solvency, since they all yield identical allocations and welfare (i.e. there is Ricardian equivalence). In contrast, with distortionary taxes, given the pre-pandemia structure of tax rates, the planner's problem is more complex because it would consider the optimal structure and time-variation of tax rates. When further restricted to time-invariant tax rates, it would consider how dynamic Laffer curves limit sustainable debt levels (see D'Erasmo et al., 2016). 


\subsection{Social Welfare \& Private Utility Gains:}

In order to compare the utility that agents derive under the SPE relative to the DCE, define $\Delta U_{i} \equiv$ $U_{i}^{S P E}-U_{i}^{D C E}$ for agents of type $i=1,2$ where $U_{1}$ and $U_{2}$ are the lifetime utility functions shown in (2) and (7). Then, denoting excess consumption levels as $\tilde{C}_{t} \equiv C_{t}-L_{t}^{\omega} / \omega$ and $\tilde{h}_{t} \equiv h_{t}-\bar{h}_{t}$ and using the results from the SPE and the DCE yields these expressions:

$$
\begin{aligned}
\Delta U_{1}= & \sum_{t=0}^{j} \beta^{t}\left[a\left(\ln \left(\tilde{C}_{t}^{s p}\right)-\ln \left(\tilde{C}^{\star}\right)\right)+(1-a)\left(\ln \left(\tilde{h}_{t}^{s p}\right)-\ln \left(\tilde{h}_{t}^{\star}\right)\right)\right] \\
+ & {\left[\sum_{t=0}^{j} \beta^{t}\left(\ln \left(\frac{\Omega^{s p}}{1+\gamma_{1}\left(\Omega^{s p}-1\right)}\right)-\ln \left(\frac{\Omega^{\star P}}{1+\gamma_{1}\left(\Omega^{\star P}-1\right)}\right)\right)\right.} \\
& \left.+\frac{\beta^{j}}{1-\beta}\left(\ln \left(\frac{\Omega^{s p}}{1+\gamma_{1}\left(\Omega^{s p}-1\right)}\right)-\ln \left(\frac{\Omega^{\star N P}}{1+\gamma_{1}\left(\Omega^{\star N P}-1\right)}\right)\right)\right], \\
\Delta U_{2}= & \sum_{t=0}^{j} \beta^{t}\left[a\left(\ln \left(\tilde{C}_{t}^{s p}\right)-\ln \left(\tilde{C}^{\star}\right)\right)+(1-a)\left(\ln \left(\tilde{h}_{t}^{s p}\right)-\ln \left(\tilde{h}_{t}^{\star}\right)\right)\right] \\
+ & {\left[\sum_{t=0}^{j} \beta^{t}\left(\ln \left(\frac{1}{1+\gamma_{1}\left(\Omega^{s p}-1\right)}\right)-\ln \left(\frac{1}{1+\gamma_{1}\left(\Omega^{\star P}-1\right)}\right)\right)\right.} \\
& \left.+\frac{\beta^{j}}{1-\beta}\left(\ln \left(\frac{1}{1+\gamma_{1}\left(\Omega^{s p}-1\right)}\right)-\ln \left(\frac{1}{1+\gamma_{1}\left(\Omega^{\star N P}-1\right)}\right)\right)\right] .
\end{aligned}
$$

Using these results, the change in social welfare $(\Delta W)$ under the SPE allocations with the optimal lockdown and transfer policies relative to the unregulated DCE allocations can be expressed as:

$$
\Delta W=\phi \gamma_{1} \Delta U_{1}+(1-\phi) \gamma_{2} \Delta U_{2}
$$

Thus, the change in social welfare attained by the optimal policies equals the valuation of the individual lifetime utility changes valued using the social welfare function.

To obtain a cardinal measure of $\Delta W$, we follow the standard procedure of expressing welfare gains in terms of a compensating variation in consumption. In particular, we calculate the percentage increase in consumption of non-health goods common across households and time periods $(\Lambda)$ that would be needed for the DCE to yield the same social welfare as under the SPE allocations. That is, we compute the value of $\Lambda$ that solves this equation:

$$
\begin{aligned}
\phi \sum_{t=0}^{\infty} \beta^{t} \gamma_{1} & \left(a \ln \left(c_{t}^{1 \star}(1+\Lambda)-\frac{\left(L_{t}^{\star}\right)^{\omega}}{\omega}\right)+(1-a) \ln \left(h_{t}^{1 \star}-\bar{h}_{t}\right)\right) \\
& +(1-\phi) \sum_{t=0}^{\infty} \beta^{t} \gamma_{2}\left(a \ln \left(c_{t}^{2 \star}(1+\Lambda)-\frac{\left(L_{t}^{\star}\right)^{\omega}}{\omega}\right)+(1-a) \ln \left(h_{t}^{2 \star}-\bar{h}_{t}\right)\right)=W^{s p}
\end{aligned}
$$

where $W^{s p}$ is given by eq. (43) evaluated at the SPE allocations. Note that, while the duration of the pandemia does not alter allocations and prices in the DCE and SPE (it only determines when the 
economy switches from the P to the NP phase), it does matter for the size of all of these individual utility and social welfare effects. In particular, the effects of the pandemia on social welfare and individual utility are larger for pandemias that last longer.

The term in the first row in the right-hand-side of equations (55)-(56) for $\Delta U_{1}$ and $\Delta U_{2}$ is the same, because it represents the aggregate effects of the planner's management of the outputpandemia tradeoff by neutralizing the utilization externality. Since, as we showed earlier, the SPE's aggregate allocations are independent of inequality, this term depends only on aggregate allocations and not on their distribution across agents. In the DCE, aggregate labor and consumption of nonhealth goods are constant at the same level in the P and NP phases, so that $\tilde{C}^{\star}$ is constant at all times. During the pandemia, however, aggregate excess health goods consumption $\left(\tilde{h}_{t}^{\star}\right)$ falls because of the increase in $\bar{h}_{t}$ for $t=0, . ., j$. The utilization externality implies that these allocations are suboptimal. Hence, during the pandemia the planner lowers the utilization rate, which reduces $\tilde{C}_{t}^{s p}$ but props-up $\tilde{h}_{t}^{s p}$. The post-pandemia phase washes out from this term, because, as explained earlier, for all $t>j$ there is no utilization externality and hence the aggregate allocations of labor, non-health output and consumption of both goods are the same in the DCE and SPE.

The second and third rows in the right-hand-side of $\Delta U_{1}$ and $\Delta U_{2}$ reflect the distributional effects, with the parts due to the P and NP phases shown in the second and third rows, respectively. $\Omega^{\star P}>\Omega^{\star N P} \geq \Omega^{s p}$ is a sufficient condition for these effects to be negative for $\Delta U_{1}$ and positive for $\Delta U_{2}$. These distributional effects are determined by a collection of constant terms that depend on $\gamma_{1}$ and the marginal utility ratios of the planner $\left(\Omega^{s p}\right)$ vis-a-vis those in the DCE $\left(\Omega^{\star P}, \Omega^{\star N P}\right) .{ }^{15}$ The terms for the pandemia phase reflect the result justifying increased transfers to type-2 agents during the pandemia, because the distribution of resources for health and nonhealth consumption is suboptimal and worsens during the pandemia (since $\Omega^{\star P}$ rises). The terms for the post-pandemia phase show that, as explained earlier, the planner redistributes resources to type-2 agents even without a pandemia (as long as $\Omega^{s p}<\Omega^{\star N P}$ ).

For quantitative analysis, expressions (55) and (56) provide an intuitive way of separating the social welfare gain into key components: First, the gains due to correcting the efficiency loss affecting aggregate allocations via the utilization externality. Second, the gains due to the socially optimal redistribution during a pandemia (which also depend partially on the utilization externality, since a larger externality implies more inequality in the DCE). Third, the gains due to redistribution even without a pandemia, because of the planner's dislike for inequality in general.

Evaluating $\Delta U_{1}$ and $\Delta U_{2}$ separately from social welfare is also helpful for assessing whether the optimal policy is Pareto efficient (i.e. $\Delta U_{1}, \Delta U_{2} \geq 0$ ). For this to be the case, the utility gain for type1 agents from correcting the aggregate effects of the utilization externality must exceed their loss due to the redistribution in favor of type-2 agents. A heuristic argument suggests that, for given social welfare weights, the SPE can be Pareto efficient if $\gamma_{1}$ is sufficiently high. Start with some $\gamma_{1}$ that yields a particular $\Omega^{* N P}\left(\gamma_{1}\right)$ and assume we set $\Omega^{s p}=\Omega^{* N P}\left(\gamma_{1}\right)$. As we increase $\gamma_{1}$ keeping $\Omega^{s p}$ fixed, the

\footnotetext{
${ }^{15}$ In the $[1, \infty)$ interval of $\Omega^{s p}$, the utilitarian planner $\left(\Omega^{s p}=1\right)$ has the strongest desire for reallocating resources. All the terms that include $\Omega^{s p}$ vanish from $\Delta U_{1}, \Delta U_{2}$, which implies that the second and third rows of $\Delta U_{1}\left(\Delta U_{2}\right)$ take their most negative (positive) values. In particular, comparing the second rows of the two expressions shows that the planner has the strongest desire to redistribute when the pandemia hits, relative to scenarios with $\Omega^{s p}>1$.
} 
utility of type- 1 agents rises (locally) because the cost of redistribution falls, since the second row of $\Delta U_{1}$ increases (becomes less negative) and the third row is zero (since $\Omega^{s p}=\Omega^{* N P}\left(\gamma_{1}\right)$ ). The result is not general, however, because the redistribution costs and $\Delta U_{1}$ are nonlinear functions of $\gamma_{1}$, but as we verify in the numerical example below, it is possible to have a paremeterization such that for given $\Omega^{s p}$ there is an interval of $\gamma_{1}$ values such that $\Delta U_{1}, \Delta U_{2} \geq 0$.

\section{Quantitative Analysis}

In this section, we study the model's quantitative predictions by examining numerical solutions based on a calibration to U.S. data.

\subsection{Calibration}

Table 4 lists the model's calibrated parameter values. The values of all of the parameters, except those of the Stone-Geary utility and the $f(m K)$ function, are easy to set following a conventional calibration approach. The model is set to a quarterly frequency with a standard discount factor of $\beta=0.99$. The Frisch elasticity of labor supply is set to 2, which is also a standard value in the literature, and since the Frisch elasticity in the model is $1 /(\omega-1)$, we obtain $\omega=1.5$. The labor share in production is set to $\alpha=0.7$, which is a common value based on historical U.S. data. Utilization is normalized so that $m=1$ without pandemia, which is equivalent to full capital utilization. The depreciation (or utilization cost) function is modified slightly to adopt a formulation typical of dynamic macro models (see Mendoza et al., 2014): $\delta\left(m_{t}\right)=\chi_{0} \frac{m_{t}^{\chi_{1}}}{\chi_{1}}$. Without pandemia, since $m_{t}=1$, the capital depreciation rate satisfies $\delta=\frac{\chi_{0}}{\chi_{1}}$, where $\delta$ is set to a depreciation rate of 0.0164 per quarter, consistent with the calibration to U.S. data in Mendoza et al. (2014) and D'Erasmo et al. (2016). The capital stock is set to $K=6.04$, which is consistent with a capital-GDP ratio of 3 . The value of $\chi_{0}$ then follows from the DCE optimality condition for capital utilization, which yields $\chi_{0}=(1-\alpha)(L(K, m) / K)^{\alpha}=0.10$, where $L(K, m)$ is the solution to eq. (25) for $K=6.04$ and $m=1$, and then the condition that $\delta=\frac{\chi_{0}}{\chi_{1}}$ yields $\chi_{1}=6.10$. Finally, $\gamma_{1}=0.2$ because the top quintile of the U.S. wealth distribution owned nearly 90 percent of the wealth in 2017 (Leiserson et al., 2019), and for simplicity we focus on the case in which $\Omega^{s p}=\Omega^{\star N P}$, so that the SPE supports the DCE without pandemia and there is no incentive to redistribute except when a pandemia hits.

To calibrate the Stone-Geary preferences, we normalize the endowment of health goods so that $H=1$. Hence, $h^{\star}$ represents the percent of the available supply of health goods that constitutes subsistence demand in normal times. The value of $h^{\star}$ is set by estimating a standard linear-expendituresystem regression of nominal expenditures of health goods and services on nominal expenditures of non-health goods and services and the price of health goods. This regression follows from the pricing condition for the NP phase, eq. (34), using the resource constraint for non-health goods and the market-clearing condition for health goods. ${ }^{16}$ The value of $h^{\star}$ corresponds to the coefficient on

\footnotetext{
${ }^{16}$ After simplifying terms, combining these expressions yields $p^{h} H=\frac{1-a}{a}\left(1-\frac{\delta(\cdot) K}{Y}-\frac{\alpha}{\omega}\right) Y+h^{\star} p^{h}$.
} 
$p_{t}^{h}$, which yields $h^{\star}=0.0948$ with a standard error of 0.0235 and a p-value of $0.0002 .{ }^{17}$

Table 4: Calibration to U.S. Data

\begin{tabular}{cll}
\hline \hline Parameter & Value & Reference \\
\hline$\beta$ & 0.99 & Standard for quarterly frequency \\
$\omega$ & 1.5 & Frisch Elasticity of labor supply equals 2 \\
$\alpha$ & 0.7 & Standard labor share \\
$K$ & 6.04 & Capital stock to match K/GDP=3 \\
$m^{\star}$ & 1 & Normalization \\
$\chi_{0}$ & 0.10 & Optimality condition for utilization with $m^{\star}=1$ \\
$\chi_{1}$ & 6.10 & 1.64\% depreciation rate, Mendoza et al. (2014) \\
$\gamma_{1}$ & 0.2 & Top quintile owns 90\% of U.S. wealth in 2017, Leiserson et al. (2019) \\
$H$ & 1 & Normalization \\
$h^{\star}$ & 0.0948 & Linear-expenditure-system regression \\
$a$ & 0.756 & Average nonhealth-to-health consumption and GDP ratios, 2009-2018 \\
$\theta$ & $0-0.1069$ & Interval that supports DCE solutions \\
\hline \hline
\end{tabular}

The share of non-health expenditures $a$ is determined by imposing on the same pricing condition (34) the estimated value of $h^{\star}=0.0948$ and the average ratios of non-health to health consumption and non-health to health GDP for the period 2009-2018, which are 5.01 and 4.73, respectively. We use 2009-2018 data because they yield stable averages for these ratios, after several years in which both fell steadily. This yields $a=0.756$.

The last item that needs to be specified is the function $f\left(m_{t} K\right)$ that maps utilization into subsistence health demand during a pandemia. As noted earlier, the function is assumed to be monotonically increasing. A concave (convex) $f(\cdot)$ would represent an economy in which reductions in utilization are less (more) effective at reducing the stress on the health system during a pandemia. For simplicity, we assume a linear function $f\left(m_{t} K\right)=\theta m_{t} K$, so that the elasticity of $\bar{h}$ with respect to $m K$ is equal to $\theta$. We know little about $\theta$, but given the value of $K$, equation (40) yields an upper bound $\tilde{\theta}$ at which health demand of type-2 agents equals their subsistence demand and there is no DCE solution with pandemia. Hence, we will study model solutions for $\theta \in[0, \tilde{\theta})$. Moreover, within this interval, we examine detailed solutions for the value of $\theta$ that makes the drop in U.S. non-health GDP observed during the pandemia consistent with an optimal lockdown. Matching the decline of 8.8 percent in U.S. non-health GDP in the second quarter of 2020 relative to the first quarter as part of the SPE solution requires $\theta=0.0918$. The corresponding utilization rate is 0.848 and hence $f(m K)=0.0918 \times 0.848 \times 6.04=0.47$. Thus, accounting for the observed non-health GDP drop as the result of an optimal lockdown implies a sharp increase in subsistence demand for health from 9.48 to $9.48+47=56.4$ percent of the available supply.

\footnotetext{
${ }^{17}$ The regression uses data for 1960-2018. Expenditures are proxied by GDP of health and non-health goods. The price index corresponds to the GDP deflator for the health sector (obtained from the BLS). Expenditures are expressed as indexes with the same base year as the deflator. Other time series used are Total National Health Expenditures, Health Investment, Health Consumption Expenditures, obtained from the National Health Expenditure database of the Centers for Medicaid and Medicare Services (CMS), and Nominal GDP and Gross Private Domestic Investment, obtained from the BLS and BEA, respectively. The regression is estimated in second differences, because non-health expenditures are integrated of order two, reflecting the sharp growth of the health sector relative to the rest of the U.S. economy.
} 


\subsection{Results}

Table 5 shows a set of results for the calibration with $\theta=0.0918$. Column (I) shows the equilibrium without pandemia, for which $\mathrm{DCE}_{N P}=\mathrm{SPE}_{N P}$ since DCE and SPE are the same in normal times. Column (IV) is the SPE solution for the pandemia that rationalizes the observed output drop as resulting from an optimal lockdown $\left(\mathrm{SPE}_{P}\right)$. Columns (II) and (III) show two DCE solutions: Column (II) is the NL case studied in Section 3, in which utilization is unaffected by the pandemia $\left(\mathrm{DCE}_{P, N L}\right)$, and Column (III) is the OL case with an ad-hoc lockdown of the same size as the optimal lockdown $\left(\mathrm{DCE}_{P, O L}\right) . \operatorname{Tr}$ is unchanged from the normal-times level in both DCE solutions. Column (I) is in levels and the rest are percent changes relative to NP levels, except $m$ and $T r / G D P$ for which we show percentage points changes and $\Omega$ and $\bar{h}$ which are always in levels.

Table 5: Competitive \& Social Planner's Equilibria for $\theta=0.0918$

\begin{tabular}{|c|c|c|c|c|}
\hline Variable & $\begin{array}{c}(\mathrm{I}) \\
\text { Normal Times } \\
\text { (levels) } \\
\mathrm{DCE}_{N P}=\mathrm{SPE}_{N P}\end{array}$ & $\begin{array}{c}(\mathrm{II}) \\
\text { No Lockdown } \\
\text { (percent changes) } \\
\text { DCE }_{P, N L} \\
\end{array}$ & $\begin{array}{c}\text { (III) } \\
\text { Observed Lockdown } \\
\text { (percent changes) } \\
\text { DCE }_{P, O L} \\
\end{array}$ & $\begin{array}{c}(\mathrm{IV}) \\
\text { Social Planner } \\
\text { (percent changes) } \\
\mathrm{SPE}_{P} \\
\end{array}$ \\
\hline \multicolumn{5}{|l|}{ Aggregate variables: } \\
\hline$\Omega^{\circ}$ & 3.46 & 16.46 & 9.54 & 3.46 \\
\hline $\bar{h}$ & 0.09 & 0.65 & 0.56 & 0.56 \\
\hline $\mathrm{GDP}^{\mathrm{NH}}$ & 2.01 & 0 & -8.84 & -8.84 \\
\hline$m$ & 1 & 0 & -15.18 & -15.18 \\
\hline$l$ & 1.26 & 0 & -5.99 & -5.99 \\
\hline$\pi$ & 0.5 & 0 & 1.85 & 1.85 \\
\hline$c$ & 1.91 & 0 & -6.02 & -6.02 \\
\hline$w$ & 1.12 & 0 & -3.04 & -3.04 \\
\hline$p^{h}$ & 0.35 & 157.76 & 101.1 & 101.1 \\
\hline \multicolumn{5}{|l|}{ Individual variables: } \\
\hline$c_{1}$ & 3.2 & 51.91 & 30.54 & -4.93 \\
\hline$c_{2}$ & 1.59 & -26.07 & -24.38 & -6.57 \\
\hline$h_{1}$ & 2.19 & -6.02 & -4.35 & -28.25 \\
\hline$h_{2}$ & 0.7 & 4.71 & 3.4 & 22.08 \\
\hline$\tilde{c}_{1}$ & 2.26 & 73.49 & 46.92 & -3.3 \\
\hline$\tilde{c}_{2}$ & 0.65 & -63.55 & -46.73 & -3.3 \\
\hline$\tilde{h}_{1}$ & 2.1 & -32.69 & -26.94 & -51.92 \\
\hline$\tilde{h}_{2}$ & 0.61 & -85.86 & -73.51 & -51.92 \\
\hline \multicolumn{5}{|l|}{ Transfers \& Welfare } \\
\hline $\operatorname{Tr} / G D P(\%)$ & 14.5 & -2.74 & -1.00 & 10.85 \\
\hline Welfare Gain (\%) & n.a. & n.a. & n.a. & $0.82(0.33)$ \\
\hline$\Delta U_{1}$ & n.a. & n.a. & n.a. & $-2.06(-1.65)$ \\
\hline$\Delta U_{2}$ & n.a. & n.a. & n.a. & $4.08(2.35)$ \\
\hline
\end{tabular}

Notes: The "Normal Times" column shows the equilibrium without pandemia (DCE and SPE are identical because the calibration assumes $\Omega^{s p}=\Omega^{\star N P}$ ). Allocations and prices in the Observed Lockdown and No Lockdown scenarios are reported as percent changes relative to Normal Times, except $\bar{h}$ and $\Omega$ are shown in levels and $m$ and $\operatorname{Tr} / G D P$ are differences in percentage points relative to their normal-times values. Welfare gains, $\Delta U^{1}$ and $\Delta U^{2}$ are as defined in the text. Welfare gains assume the pandemia lasts four quarters and are relative to the No Lockdown scenario with values in parenthesis relative to the Observed Lockdown scenario.

The aggregate allocations for $\mathrm{DCE}_{P, O L}$ and $\mathrm{SPE}_{P}$ show that the 8.8 percent output drop during the pandemia is associated with a cut in $m$ of 15 percentage points and declines in consumption 
and labor of about 6 percent. For the planner, the cut in $m$ is the optimal response to the utilization externality. Profits of the non-health sector rise 1.9 percent because of reduced utilization costs. Subsistence health demand climbs from 0.09 to 0.56 , as explained earlier, and the relative price of health goods rises 101.1 percent, because aggregate excess consumption of health goods falls much more than for non-health goods. Without lockdown, the results for aggregate allocations in the $\mathrm{DCE}_{P, N L}$ differ from the NP phase only in that the relative price rises sharply, by nearly 158 percent. This is larger than in the OL and planner's solutions because there is no cut in utilization moderating the spike in subsistence health demand, which climbs to 0.65 instead of 0.56 .

Regarding individual allocations, recall that the planner has weights set so as to match the ratio of excess consumptions across agents without pandemia $\left(\Omega^{s p}=3.46\right)$. Hence the planner cuts excess consumptions of each agent by the same percentage relative to the NP state $(-3.3$ and -51.9 percent for non-health and health goods, respectively). In levels, however, the planner reduces entrepreneurs' consumption of both goods $(-4.9$ and -28.3 percent for non-health and health goods, respectively), while for workers it reduces non-health consumption by -6.6 percent but increases health consumption by 22.1 percent. The planner spreads the drop in the aggregate supply of nonhealth goods triggered by the optimal lockdown relatively evenly across agents, while the contrast of the large drop it assigns to health consumption for entrepreneurs v. the large increase for workers is in response to the planner's strong incentive to redistribute so as to keep $\Omega^{s p}=3.46$. This redistribution requires an increase in the ratio of transfers to GDP of nearly 11 percentage points, which would be even larger without the strong valuation effect driving up the relative value of health GDP. Assuming the pandemia lasts four quarter, the optimal lockdown and transfers policies increase welfare by 0.82 percent, which is a sizable gain.

Inequality worsens sharply in the NL scenario, with $\Omega_{N L}^{* P}$ rising to 16.5 . This large increase in excess consumption inequality results from $\tilde{c}$ increasing (falling) by $73.5(-63.6)$ percent for type-1 (type-2) agents. Since labor supply and labor disutility are the same for both agents, this implies that nonhealth consumption also rises sharply for type- 1 agents and falls sharply for type-2 agents. Regarding excess health consumption, $\tilde{h}$ falls much less for type- 1 than type- 2 agents $(-32.7 \mathrm{v} .-85.9$ percent, respectively). Hence, the pandemia moves workers closer to the subsistence demand for health at a much faster pace than entrepreneurs. Income inequality also worsens sharply as result of the large increase in the value of the endowment of health goods that type- 1 agents own. Transfers are unchanged in levels from the NP state, but since the value of GDP rises with $p^{h}$, transfers as a share of GDP fall by -2.7 percentage points.

The OL equilibrium with the ad-hoc lockdown (Column (III)) performs better than the NL case but it is still inferior to the optimal policy scenario. The ad-hoc lockdown yields the same aggregate allocations and prices as for the planner. Importantly, it also moderates the adverse effects of the pandemia on inequality. The ratio of excess consumptions rises to $\Omega_{O L}^{* P}=9.5$, instead of 16.5 in the NL case, and this is possible because both $\bar{h}$ and $p^{h}$ increase less. The latter implies that income inequality also worsens less in the OL than the NL case. ${ }^{18}$ The implied weaker valuation effect also

\footnotetext{
${ }^{18}$ Note that wage income falls by the same amount for both agents, since they supply the same labor at the same wage, and that the small increase in profits worsens income inequality but it is dwarfed by the effect of the smaller hike in $p^{h}$.
} 
implies that transfers (which are constant at the NP level) fall less as a share of GDP, by 1 percentage point instead of 2.7. The SPE yields a welfare gain of 0.33 percent relative to this OL case, and since the gain relative to the $\mathrm{NL}$ case was 0.82 , it follows that the lockdown alone yields a welfare gain of about 50 basis points and the transfers add 32. Thus, roughly $3 / 5$ ths of the total welfare gain produced by the optimal policies is due to the lockdown. Keep in mind, however, that the lockdown has effects both on aggregate efficiency (by tackling the utilization externality) and on inequality (by moderating the hikes in $\bar{h}$ and $p^{h}$ ). Thus, the ad-hoc lockdown does help mitigate the adverse inequality effects of the pandemia but not nearly enough as is socially optimal.

The results in Table 5 are for $\theta=0.0918$, which was targeted to match the observed 2020Q2 fall in U.S. non-health GDP. We acknowledge, however, that there is substantial parameter uncertainty regarding the value of $\theta$. Hence, we turn now to examine the full spectrum of solutions for the interval of $\theta$ values that support competitive equilibria. As noted earlier, this is possible for $\theta \in[0, \tilde{\theta})$ where $\tilde{\theta}$ is the upper bound at which health consumption of workers hits the subsistence level. Under the calibration to U.S. data, the DCE with no lockdown yields $\tilde{\theta}=0.1069$.

Figure 4 shows utilization and non-health GDP. The blue curves show the SPE solutions, the black lines show the DCE under the NL case (for which aggregate allocations are invariant in $\theta$ ), and the red lines show the OL solutions for the DCE with an ad-hoc lockdown of the same size as the optimal lockdown that matches the drop in U.S. non-health GDP. The black and red dots denote the solutions shown in Table 5. By construction, the red dots must be at the intersection of the blue curves with the red lines (i.e., an ad-hoc lockdown of the same size as the optimal lockdown). The dashed, red vertical lines identify $\tilde{\theta}$. The DCE lines are discontinuous at that point because there is no DCE solution when $\theta=\tilde{\theta}$.

Figure 4: Utilization and Non-Health Output in Pandemia as $\theta$ Varies

Utilization

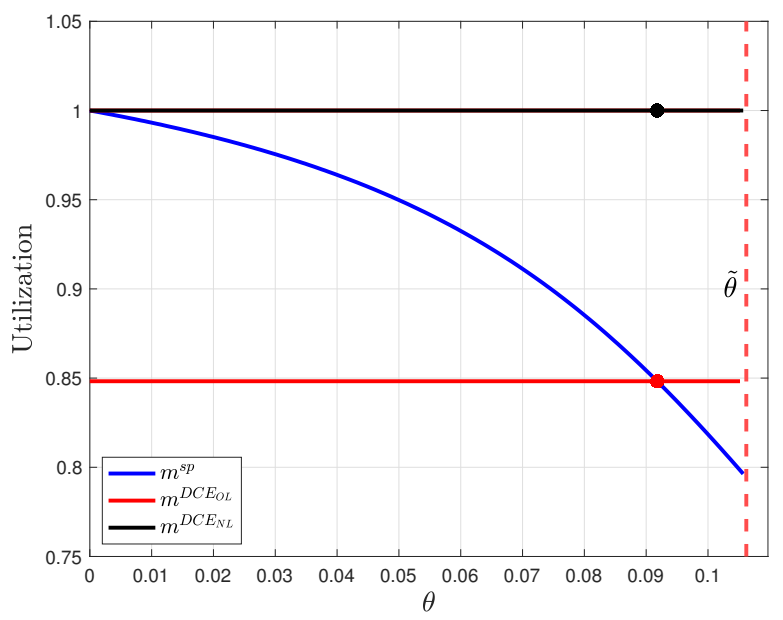

Non-Health GDP

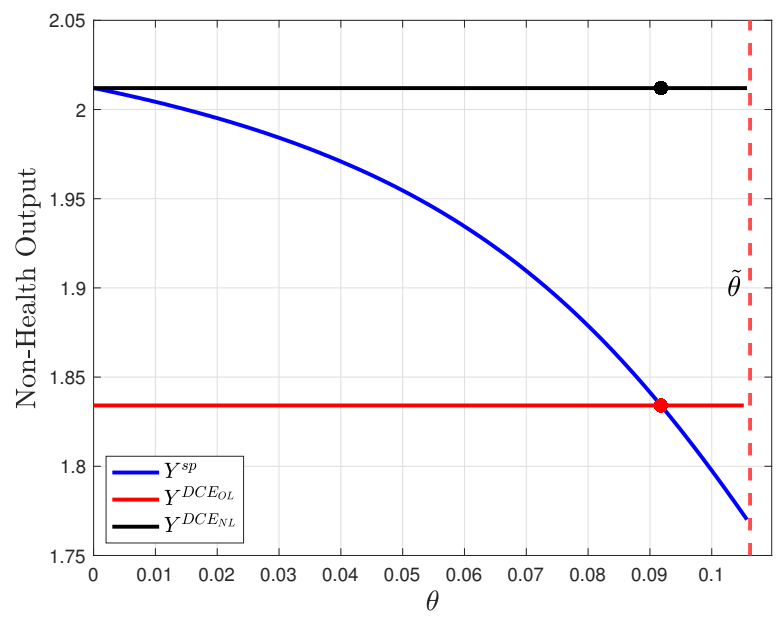

Figure 4 shows that the planner's optimal reductions in utilization and non-health GDP are concave in $\theta$. Hence, as the elasticity of subsistence health demand to utilized capital rises, the optimal lockdown in response to a pandemia increases with $\theta$ at a faster rate. Scenarios with low $\theta$ s are un- 
likely to be relevant. They represent weak pandemias for which $\bar{h}$ varies little in response to cuts in $m$ (i.e., social distancing is not that important to alter contagion) and as a result small cuts in utilization and output, namely weak lockdowns, would suffice to address the utilization externality. This is captured in the Figure by the small gap between the black lines that correspond to the NL solutions and the blue curves of the planner's solutions for $\theta<0.05$.

Pandemias become relevant as $\theta$ rises above 0.05 . In this region, the concavity of the planner's choices has a key implication: Small "errors" in measuring $\theta$ result in non-trivial errors in the utilization and output cuts adopted to respond to a pandemia. The gaps between the blue and red curves illustrate how these errors vary as the "true" value of $\theta$ varies if the lockdown that would be optimal for $\theta=0.0918$ is adopted. If $\theta$ is in fact slightly smaller (larger) the lockdown would be too strict (weak) and non-health GDP would be allowed to fall too much (little) relative to what is truly optimal. For example, if $\theta=0.08$, the ad-hock lockdown of the OL line would cut utilization and output by 3.2 and 2.3 percentage points more than what is optimal, respectively. Relative to pre-pandemia levels, utilization and output would fall by 15.2 and 8.8 percentage points, respectively, compared with optimal drops of 12 and 6.5 percentage points each.

An alternative interpretation of Figure 4 is as indicative of the implications of cross-country or cross-region heterogeneity in health systems and other relevant pre-pandemia conditions (like income per capita, life expectancy, etc.). Countries with weaker pre-pandemia conditions can be viewed as countries with higher $\theta$, and hence faced with a pandemia they require larger optimal lockdowns which imply larger output drops. The relative size of the health sector also captures cross-country differences in health systems. Equation (48) implies that the utilization externality is weaker in countries where $H$ is larger, and hence for the same $\theta$ the optimal lockdown and output drop would be smaller in these countries. The same applies to countries where $a$ is larger.

The two plots in Figure 5 show how the rise in $p^{h}$ and the worsening consumption inequality due to a pandemia vary with $\theta$ in the SPE (in blue) and in the DCE cases for no-lockdown (in black) and the ad-hock lockdown (in red) that matches the observed decline in non-health GDP. Black and red dots denote again the NL and OL outcomes in Table 5 . The planner chooses higher prices for $\theta$ values that would make the optimal lockdown of Table 5 excessive $(\theta<0.0918)$, and lower prices when the opposite occurs. In contrast, the SPE price hikes are always smaller than those for the no-lockdown DCE case, because the planner reduces utilization and this moderates the increase in the relative price. Prices are nearly linear in $\theta$ for the planner but they are convex for both DCE cases, and hence small errors in assessing the value of $\theta$ to implement lockdowns would result in large differences in price hikes during pandemias.

The price hikes are quite large overall, except for $\theta$ values that result in negligible drops in utilization and output. In line with the argument that pandemias are weak for $\theta<0.05$, prices in the no-lockdown DCE scenario are negligibly different from those produced under the optimal policies for those $\theta$ values. For $\theta>0.05$, the optimal policy yields price hikes of at least 45 percent and as much as 105 percent. Price hikes in the no-lockdown DCE case are uniformly higher, ranging from 50 to 230 percent. Hence, the model predicts large relative price movements during pandemias.

The graph for the ratios of excess consumption (plotted in a logarithmic scale) shows a constant 
$\Omega^{s p}$ for the planner, which follows from the welfare weights calibrated to match the value of $\Omega$ in the DCE without pandemia (3.46). During the pandemia, consumption inequality is sharply higher in the DCEs for the NL and OL scenarios than for the planner, and it worsens at an increasing rate as $\theta$ rises driven by the rising $p^{h}$ and the worsening income inequality. For $\theta$ ranging between 0.05 and 0.1 , the values of $\Omega$ in the NL (OL) scenario are in the 6-37 (5-12.5) range, much larger than the 3.46 ratio in normal times. Consumption inequality is higher in the NL than the OL case because the NL case lacks the effect of the ad-hoc lockdown moderating the increase in $p^{h}$ and the rise of income inequality in the OL case.

The left panel of Figure 6 shows the optimal transfers and the social welfare gain of the optimal lockdown and transfers policies as $\theta$ varies. Transfers are plotted as the change in the transfers-GDP ratio relative to normal times $(\triangle T r / G D P)$ and welfare is measured relative to the no-lockdown DCE assuming a 4-quarter pandemia. $\triangle \operatorname{Tr} / G D P$ rises monotonically because consumption inequality increases with $\theta$ (see Figure 5) and strengthens the incentives to redistribute income and consumption across agents so as to maintain the ratio of excess consumptions at $\Omega^{s p}=3.46$. $\Delta \operatorname{Tr} / G D P$ is small for weak pandemias but for $\theta>0.05$ increases in transfers from 5 to 13 percentage points of GDP are optimal. ${ }^{19}$ Similarly, weak pandemias yield negligible welfare gains from implementing the optimal policies, but for $\theta>0.05$ the welfare gains are a sharply convex function of $\theta$ and grow infinitely large as $\theta$ approaches $\tilde{\theta}$, because at that point workers hit the Inada condition for health consumption in the Stone-Geary preferences. This plot also shows that the nonlinear effects of implementing policies with measurement error in $\theta$ discussed earlier have nonlinear welfare implications. For instance, around $\theta=0.0918$, which is the value that renders the observed GDP decline consistent with the optimal policy, if the "true" $\theta$ is slightly lower (higher) the welfare gain is much smaller (larger).

Figure 5: Relative Prices and Consumption Inequality in Pandemia as $\theta$ Varies

\section{Relative Price of Health Goods}

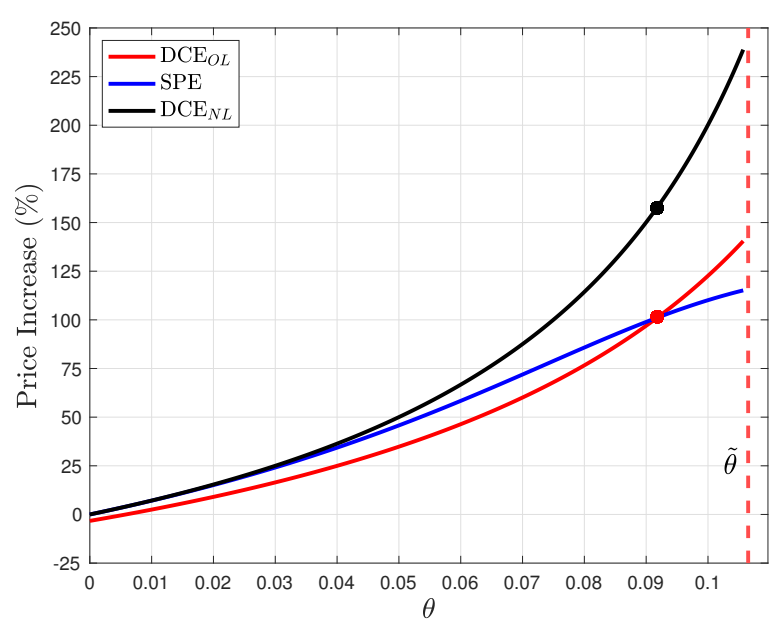

Excess Consumption Ratios

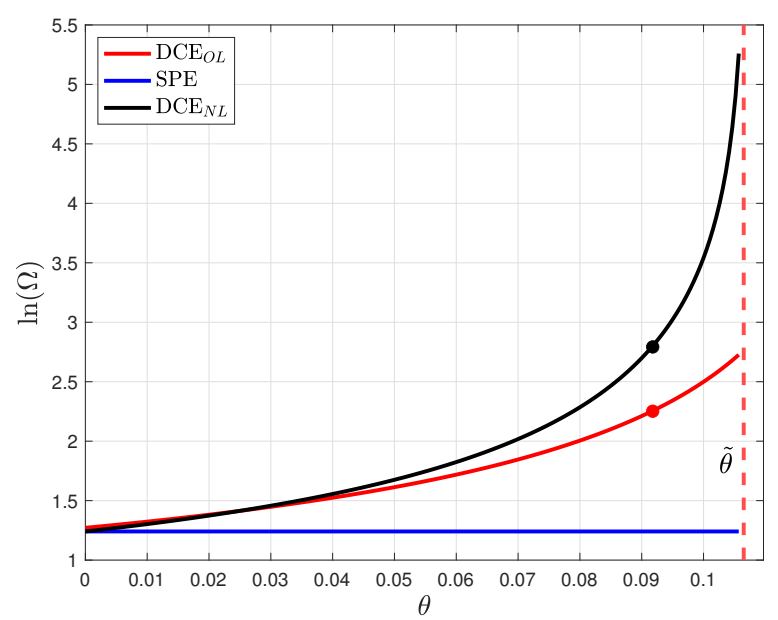

\footnotetext{
${ }^{19}$ Recall that $\Delta \operatorname{Tr} / G D P$ captures both the effect of the exogenous change in transfers and the endogenous response of GDP to the optimal policies. The valuation effect of higher $p^{h}$ reduces the ratio.
} 
The right panel of Figure 6 shows a decomposition of the welfare gains in terms of the fractions due to changes in aggregate allocations and redistribution across agents. The contribution of removing the inefficiency in aggregate allocations is always much smaller than the contribution due to redistribution, which highlights again the relevance of the effects of pandemias on inequality in the model. As $\theta$ rises, the contribution from changes in aggregate allocations shrinks and that from redistribution rises, because inequality is also increasing in $\theta$. The aggregate inefficiencies account at most for about 11 percent of the welfare gains, and that is for very small welfare gains corresponding to weak pandemias. Redistribution accounts for 88 to 96 percent of the welfare gains. This is again because type- 2 agents move closer to their subsistence level of health consumption as $\theta$ rises and as this happens they approach the Inada condition that makes the marginal utility of allocating health consumption to them infinitely large. For the optimal policy reported in Table 5, the contribution of redistribution is close to 92 percent. This result is not inconsistent with the previous finding showing that for that same optimal policy the gains from reducing $m$ are larger than those from increasing $\mathrm{Tr}$, because, as noted earlier, reducing $m$ contributes to both improve aggregate efficiency and reduce inequality.

Figure 6: Transfers \& Welfare Gains as $\theta$ Varies

Transfers and Welfare Gains

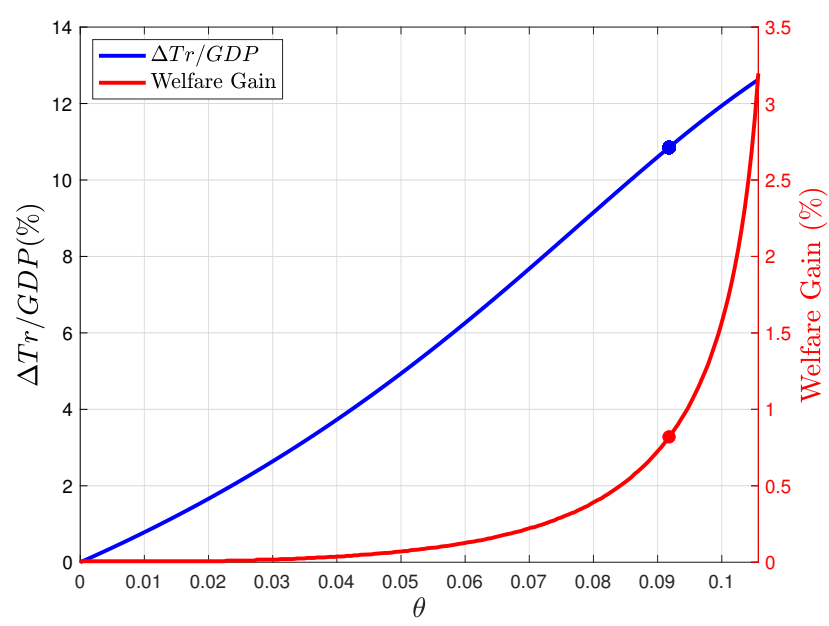

Composition of Welfare Gains

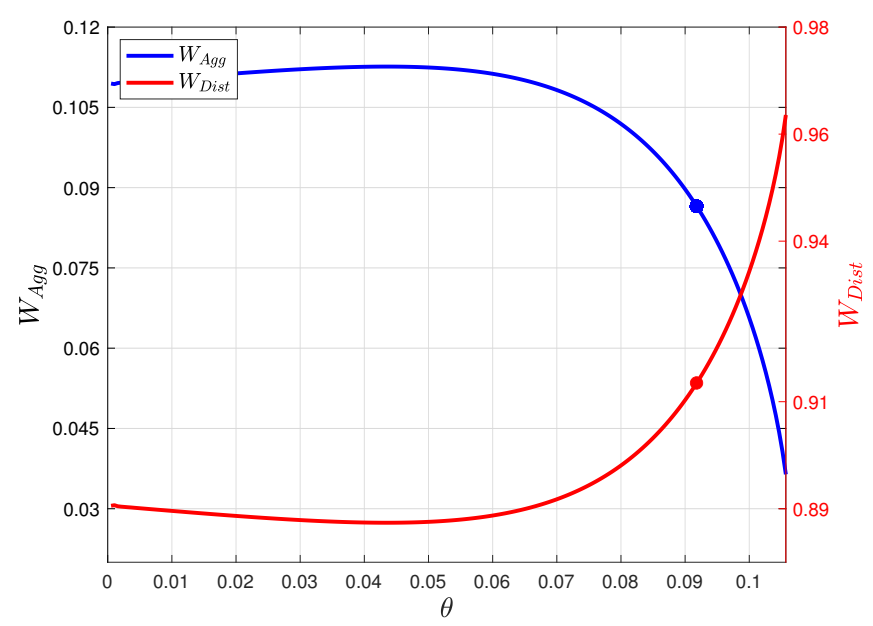

The role of inequality in these results can be illustrated further by examining the welfare implications of agent heterogeneity. Figure 7 compares social welfare gains of the optimal policies (relative to the no-lockdown DCE) as $\theta$ varies for the calibrated economy with $\gamma_{1}=0.2$ and the comparable representative-agent economy with $\gamma_{1}=1$. In the latter, the welfare gains are only due to the removal of the utilization externality. The DCE (SPE) aggregate allocations are the same as in the DCE (SPE) with two agents. Both welfare gains display the convex, asymptotic behavior as $\theta$ reaches $\tilde{\theta}$. The value of $\tilde{\theta}$, however, is about 60 percent bigger in the representative-agent model that has no inequality ( since $\gamma_{1}=1$ ). This occurs because, as explained earlier, the pandemia moves workers 
toward their subsistence health demand at a much faster pace than entrepreneurs, and hence the health system saturates at lower $\theta$ when inequality is present. At values of $\theta$ for which both models can be solved, the welfare gains of the optimal policies are negligible for the representative-agent model. A much stronger utilization externality, driven by higher $\theta$ values (above 0.14 ), would be needed in order to yield non-trivial welfare gains. At those values, however, the model would predict much larger falls in output than what has been observed (since the higher $\theta$ values would yield much larger utilization cuts).

Figure 7 also indicates that a pandemia of identical characteristics in terms of the elasticity of health subsistence to utilized capital is much more damaging for countries with higher levels of wealth inequality pre-pandemia (lower $\gamma_{1}$ ). The welfare implications of wealth inequality are also nonlinear, because the upper bound $\tilde{\theta}$ at which the health system saturates and the welfare gains of the optimal policies grow infinitely large is decreasing in $\gamma_{1}$.

Figure 7: Welfare Gains as $\theta$ Varies with Representative and Heterogeneous Agents

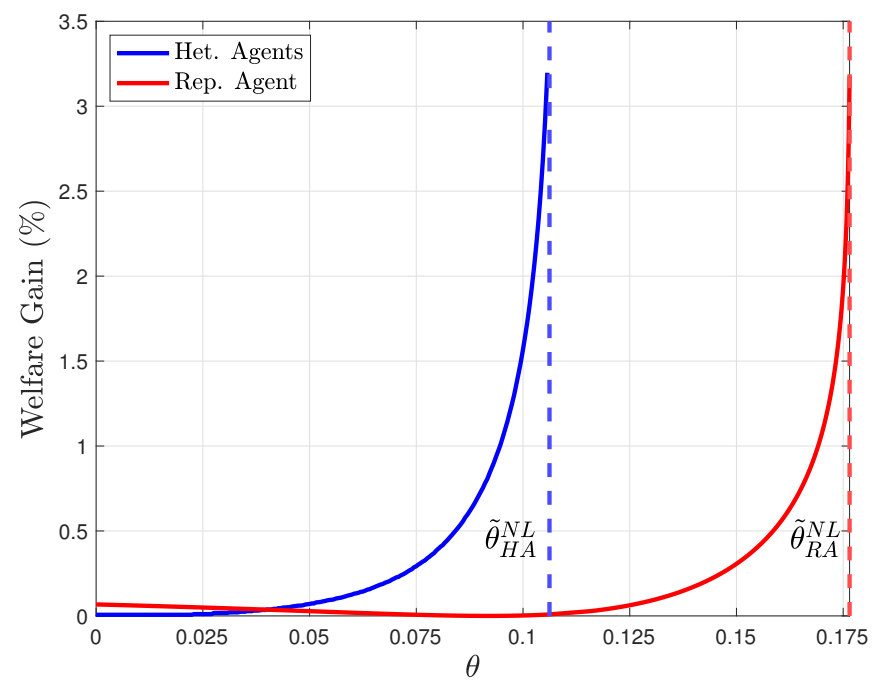

Notes: Welfare gains are computed relative to the no-lockdown DCE .

We discussed the implications of suboptimal policies resulting from parameter uncertainty or country heterogeneity related to the values of $\theta, H$ and $a$. We consider next policy errors due to adhoc deviations from the optimal transfers and lockdown policies that could be the result of political economy considerations, institutional flaws, or other frictions outside the model.

Figure 8 shows welfare costs from policies that deviate from the planner's optimal policies in the calibrated model (i.e., those reported in Table 5). To construct this Figure, we solve the DCE for arbitrary pairs of mandated utilization rates and increases in transfers, calculate the welfare gain that would be obtained by shifting to the planner's optimal policies, and plot the negative of that gain as the welfare cost of each arbitrary policy pair. The transfers policies span the $0-28 \%$ interval, defined in terms of increases relative to transfers in normal times in percent of the GDP of normal times $\left(\Delta \operatorname{Tr} / G D P_{N P}\right)$. We use a common value of GDP to scale $\operatorname{Tr}_{P}$ and $\operatorname{Tr}_{N P}$ in order to isolate 
the change in transfers per-se (which is the policy instrument) from endogenous changes in GDP. The Figure shows curves for welfare costs as a function of $\Delta T r / G D P_{N P}$ for four utilization rates $m=0.6,0.7,0.85,1$. The percent drops in non-health GDP associated with these utilization rates are $25,18.2,8.8$ and 0 , respectively. The curves are discontinuous because transfers that are too large would make excess consumption of type- 1 agents negative. The SPE's optimal policies correspond to $m^{s p}=0.85$ and $\Delta \operatorname{Tr} / G D P_{N P}{ }^{s p}=12.7 \%$. By construction, the maximum value for the curve corresponding to $m=m^{s p}=0.85$ is $\Delta \operatorname{Tr} / G D P_{N P}{ }^{s p}=12.7 \%$ and there is zero welfare cost, because this point in the curve matches the SPE. Any deviation from this policy pair reduces welfare. As before, the red and blue dots correspond to the DCE solutions for the NL and OL cases in Table 5. The horizontal line identifies the welfare cost if there is no policy change when the pandemia hits.

Figure 8: Welfare Costs of Deviating from Optimal Policies

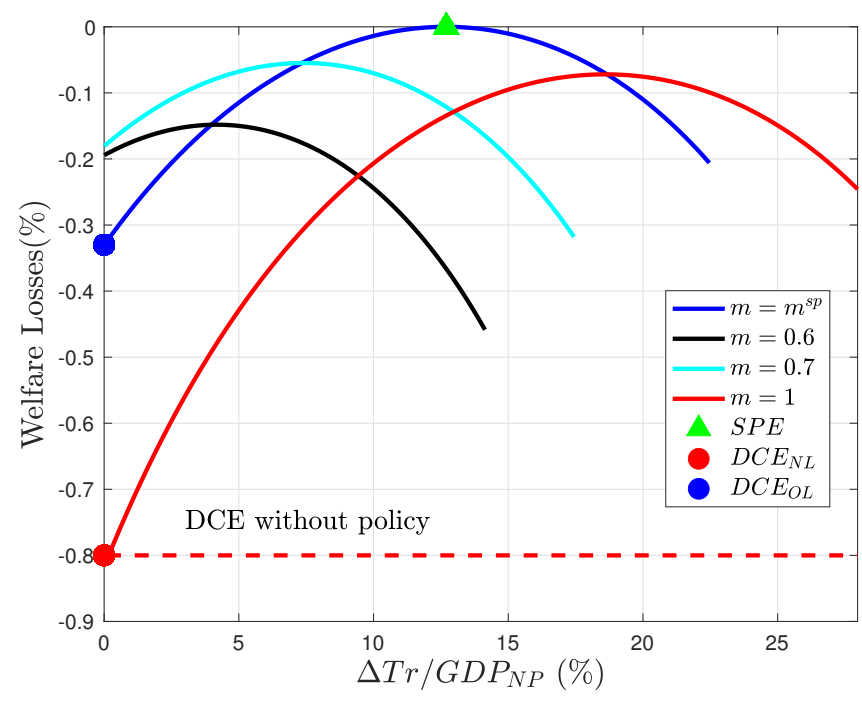

Note: The associated drops in nonhealth output with respect to the no-pandemia level are $8.84 \%$ for $m=m^{s p}, 18.2 \%$ for $m=0.7$ and $25 \%$ for $m=0.6$.

This Figure yields two important results. First, deviating from the optimal policies can have nontrivial welfare costs but, for the set of policy pairs considered, policy intervention is always preferable to no intervention and mostly by a sizable margin. Relative to the DCE without policy intervention, welfare is at least 0.35 percent higher with all policy pairs except those with no lockdown $(m=1)$ and small transfer hikes (below 5 percentage points). ${ }^{20}$ Note, however, that the ranking of the policies is not monotonic, as the crossing of the curves indicates: With low transfers, stricter lockdowns are better but as transfers increase stricter lockdowns are undesirable.

The second result is that transfers and lockdowns can be traded off widely at a small welfare cost. For instance, a no-lockdown policy with a transfers hike of 18 percentage points yields about the same welfare as one pairing a hike in transfers of about 5 percentage points with a strict lockdown

\footnotetext{
${ }^{20}$ Policies that reduce welfare below the DCE without policy changes require unrealistically large lockdowns $(m \leq 0.3)$.
} 
that reduces $m$ to 0.7 and output by 25 percent, and both policy pairs are only about 0.08 percent below the SPE in terms of welfare. Even in a scenario in which only one instrument can be used, a hike in transfers of 18 percentage points without a lockdown is only about 0.1 of percent better than a lockdown setting $m=0.6$ without increasing transfers. This is possible because either a large increase in transfers or a strict lockdown reduce the strong adverse effects on inequality caused by the pandemia. For the same reason, policies that combine weak lockdowns with small hikes in transfers are undesirable and the interaction of the two is nonlinear. For instance, the welfare loss resulting from changing from the optimal lockdown to no lockdown (i.e., the gap between the blue and red curves) grows larger as the size of the increase in transfers is reduced. This is again because both less strict lockdowns and weaker transfers programs allow the pandemia to worsen inequality more.

These results have important policy implications, because the data show that countries with high income per-capita have implemented much larger transfers policies and stricter lockdowns in response to COVID-19 than those with lower income. Data from the IMF Fiscal Monitor show that, through September 2020, the average increase in transfers for advanced economies reached 9.9 percent while for emerging and less developed countries the averages were 4.4 and 3 percent, respectively. ${ }^{21}$ For the 53 countries included in the Bloomberg resilience indicator, the log of income per-capita has a correlation with the community mobility measure of -0.2 while the correlation with Covid-related transfers is 0.5 . Hence, on average, poorer countries responded to COVID-19 with both weaker lockdowns and smaller fiscal interventions, which is the worst combination in the model.

The last graph, Figure 9, shows an important result regarding Pareto efficiency of the optimal policies. The plot shows how the lifetime utilities of type- 1 and type- 2 agents change under the SPE vis-a-vis the no-lockdown DCE as the fraction of type- 1 agents rises. For each value of $\gamma_{1}$, we obtain the SPE and no-lockdown DCE solutions, keeping social welfare weights and $\theta$ at the levels used in Table 5 (i.e., $\Omega^{s p}=3.46$ and $\theta=0.0918$ ), and we plot $\Delta U_{1}$ and $\Delta U_{2}$. At the calibrated value of $\gamma_{1}=0.2$, the SPE yields $\Delta U_{1}<0$ and $\Delta U_{2}>0$. However, if $\gamma_{1}$ is slightly higher so that it falls within the shaded area in the Figure, both agents are better off under the SPE. Thus, as suggested in the previous section, given social welfare weights, the SPE can be Pareto efficient if $\gamma_{1}$ is sufficiently high so as to reduce the per-agent cost of redistribution for type-1 enough to make them better off but also not too high so that redistribution is insufficient to make type- 2 agents better off. The per-agent cost of redistribution for type- 1 agents falls with $\gamma_{1}$ because there are more agents of this type to share the cost and the SPE allocates less consumption of health and non-health goods to type-2 agents as $\gamma_{1}$ rises (see equations (49)-(50)).

\footnotetext{
${ }^{21}$ These data include additional and accelerated spending plus foregone and deferred revenue and exclude business liquidity support (equity injections, loans, asset purchases, debt assumptions, guarantees and quasi-fiscal operations).
} 
Figure 9: Lifetime Utility Changes and Fraction of Type-1 Agents

(SPE relative to no-lockdown DCE)

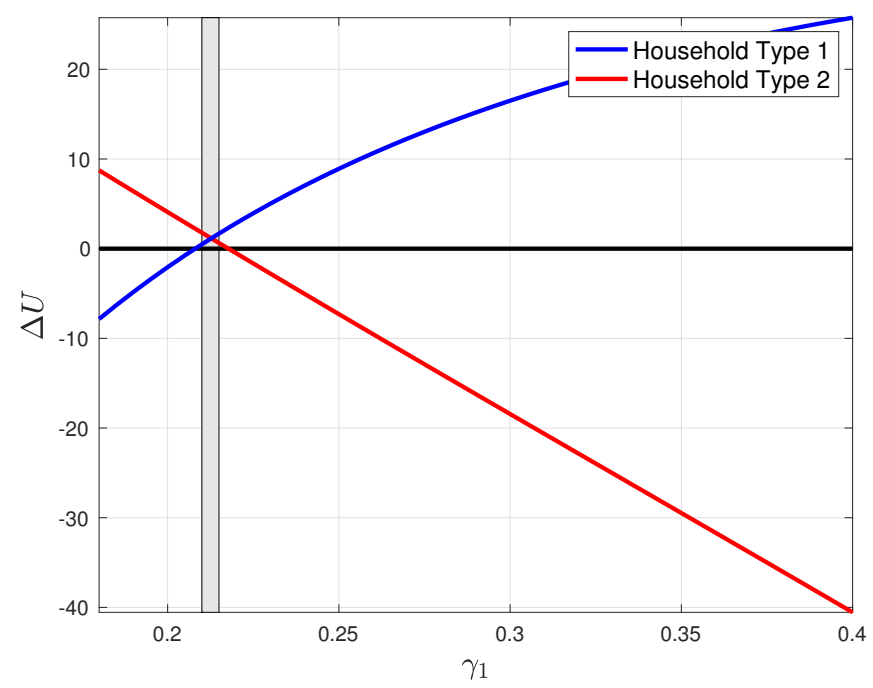

Notes: Utility changes under the SPE allocations relative to the no-lockdown DCE scenario.

\section{Conclusions}

This paper proposed a model of the macroeconomic effects of pandemias in which the saturation of the health system is the key driving force. This approach is motivated by evidence we provided on resouce shortages and capacity constraints of hospitals, sharp increases in the relative prices of key health goods and services, spikes in excess mortality beyond that explained by COVID-19, and a cross-country analysis showing that proxies for healtcare system saturation and the stringency of lockdowns are significant determinants of differences in the size of GDP drops caused by COVID-19, even after controlling for the effects of COVID infection and mortality.

Healthcare saturation is modeled by introducing Stone-Geary preferences with a jump in the subsistence demand for health goods and services during pandemias that is positively related to capital utilization. The model features entrepreneurs and workers in order to capture the effects of pandemias and lockdowns (i.e., mandated reductions in utilization) on consumption and income inequality. An output-pandemia tradeoff emerges because firms do not internalize that reducing utilization during a pandemia moves the healthcare system away from its saturation point. The pandemia moves workers closer and faster to the subsistence demand for health than entrepreneurs and it causes a sharp increase in the relative price of health goods and in the excess consumption ratio of entrepreneurs relative to workers. Lockdowns mitigate these adverse effects on inequality by mitigating the shock on subsistence health demand and its impact on the relative price of health. A planner with a standard social welfare function reduces utilization (to tackle the utilization externality) and redistributes consumption and income from entrepreneurs to workers (to keep the excess consumption ratio unchanged). Hence, the optimal policy that decentralizes the planner's allocations includes a lockdown and increased transfers to workers. 
We examined the quantitative predictions of the model using numerical solutions for a calibration to U.S. data. Two key pieces of this calibration related to the subsistence demand for health are its level in normal times and its elasticity with respect to utilized capital. The former was determined using a linear-expenditure-system regression with pre-COVID-19 data, and for the latter we examined results for the interval of elasticity values that support competitive equilibria, since the elasticity has an upper bound at the level that drives workers to reduce their demand for health to the subsistence level. Within this interval, we also studied a set of results for which the elasticity is such that the observed decline in U.S. non-health GDP results from an optimal lockdown, which requires an elasticity of 0.09 . This planner's solution was then compared with competitive equilibria in which policies are unchanged (the no-lockdown, NL, case) and in which a lockdown equal to the optimal one is implemented but transfers remained unchanged (the observed-lockdown, OL, case).

The results are indicative of the potential relevance of the proposed approach to study pandemias as a problem of health-system saturation and resource shortages and shed light on the challenges facing the design of lockdown and transfer policies to deal with pandemias. The effects of the pandemia on both aggregate efficiency and inequality are significant. For the scenario that rationalizes the observed output drop as an optimal policy, the welfare gains relative to the NL and OL cases are 0.82 and 0.33 percent, respectively. The optimal policy requires a cut in utilization of 15 percentage points (which yields a non-health output drop of 8.8 percent) and an increase in the transfers-GDP ratio of 10.9 percentage points. The relative price of health rises 101 percent under the optimal policies and the OL case, and 158 percent in the NL case. Inequality worsens very sharply during the pandemia, with the excess consumption ratio increasing by factors of 4.8 and 2.8 in the NL and OL cases, respectively. The difference between the two shows that lockdowns have strong effects on inequality, because even without transfers, a lockdown reduces the hike on subsistence health demand, which reduces the rises in relative prices and the excess consumption ratio.

Examining the set of solutions for the entire interval of feasible elasticities of subsistence health demand to utilized capital shows that the effects of pandemias on macro aggregates and inequality start to become relevant at elasticities higher than 0.05 . The output-pandemia tradeoff yields concave, negative relationships between either the planner's optimal utilization or non-health output and that elasticity. Relative prices and excess consumption ratios in the NL and OL solutions, and the welfare gains under the optimal policies are increasing, convex functions of the elasticity. Hence, small measurement error in the value of this elasticity results in non-trivial differences on the magnitude of optimal lockdown and transfer policies and their effects.

The planner undoes the large negative effect of the pandemia on inequality through the direct effect of the transfers and the indirect effect of the lockdown (which mitigates the relative price hike and the rise of the excess consumption ratio). The two effects combined contribute over 90 percent of the welfare gains of the optimal policies. The aggregate effect of the lockdown removing the utilization externality accounts for the other 10 percent. Inequality also makes the model more plausible. A planner in a representative-agent version of the model only gains by removing the utilization externality and thus needs larger elasticities of subsistence health demand to utilized capital (above 0.13) in order to yield nontrivial welfare gains. But these elasticities would yield 
unrealistically large output drops.

Deviating from the optimal policies has nontrivial welfare costs. However, policy intervention is preferable to no intervention for a large set of lockdown and transfer policy pairs. Moreover, transfers and lockdowns can be traded off widely at a small welfare cost, because either a large increase in transfers or a strict lockdown reduce the strong adverse effects of the pandemia on inequality. For the same reason, policies that combine weak lockdowns with small hikes in transfers are the worst choice. This result has important policy implications, because emerging and least developed countries responded to COVID-19 with both weaker lockdowns and smaller fiscal interventions than advanced economies. Income per capita has a correlation with lockdown effectiveness of roughly -0.2 whereas its correlation with Covid-related transfers is 0.5 . The mean increase in transfers in advanced economies has been at least 2.25 times larger than in emerging and least developed countries.

Our results also have important implications for the analysis of cross-country or cross-region responses to COVID-19. The model predicts that a pandemia is more damaging for countries with higher wealth inequality and/or weaker health systems or other pre-pandemia conditions (e.g., income per capita, life expectancy, etc.). Weaker pre-pandemia conditions can be viewed as implying higher elasticities of subsistence health demand to utilized capital which imply larger optimal lockdowns and output drops. The relative size of the health sector also captures cross-country differences in health systems. For a given elasticity, the model predicts weaker effects of pandemias in countries with larger health sectors or larger shares of non-health expenditures.

This study is a first step in a research agenda exploring the saturation of the healthcare system as the mechanism driving macroeconomic models of pandemias. The model we presented is streamlined with the intent of highlighting the essential elements of this mechanism, leaving for further research enriching the model to explore dynamic and cross-country propagation, particularly in models with capital accumulation and financial frictions, and to study the interaction of optimal lockdown and transfer policies with optimal taxation and public debt sustainability. 


\section{References}

Acemoglu, D., Chernozhukov, V., Werning, I., and Whinston, M. D. (2020). 'A Multi-Risk SIR Model with Optimally Targeted Lockdown'. NBER Working Paper No 27102.

Ajao, A., Nystrom, S., Koonin, L., Patel, A., Howell, D., Baccam, P., Lant, T., Malatino, E., Chamberlin, M., and Meltzer, M. (2015). 'Assessing the Capacity of the Healthcare System to Use Additional Mechanical Ventilators During a Large-scale Public Health Emergency'. Disaster Medicine and Public Health Preparedness, vol. 9, no. 6, 634-641.

Alvarez, F. E., Argente, D., and Lippi, F. (2020). 'A Simple Planning Problem for COVID-19 Lockdown'. NBER Working Paper No 26981.

Arellano, C., Bai, Y., and Mihalache, G. P. (2020). 'Deadly Debt Crises: COVID-19 in Emerging Markets'. NBER Working Paper No 27275.

Atkeson, A. (2020). 'What Will Be the Economic Impact of COVID-19 in the US? Rough Estimates of Disease Scenarios'. NBER Working Paper No 26867.

Azzimonti, M., Fogli, A., Perri, F., and Ponder, M. (2020). ‘Pandemic Control in ECON-EPI Networks'. NBER Working Paper No 27741.

Baqaee, D., Farhi, E., Mina, M., and Stock, J. (2020). 'Reopening Scenarios'. NBER Working Paper No 27244.

Bloom, D. E., Kuhn, M., and Prettner, K. (2020). 'Modern Infectious Diseases: Macroeconomic Impacts and Policy Responses'. NBER Working Paper No 27757.

Bodenstein, M., Corsetti, G., and Guerrieri, L. (2020). 'Social Distancing and Supply Disruptions in a Pandemic'. Finance and Economics Discussion Series 2020-031.

Britton, T. (2010). 'Stochastic Epidemic Models: A Survey'. Mathematical Biosciences, vol. 225, 24-35.

Cakmakli, C., Demiralp, S., Kalemli-Ozcan, S., Yesiltas, S., and Yildirim, M. A. (2020). 'COVID-19 and Emerging Markets: An Epidemiological Model with International Production Networks and Capital Flows'. NBER Working Paper No 27191.

Céspedes, L. F., Chang, R., and Velasco, A. (2020). 'Macroeconomic Policy Responses to a Pandemic'. Working Paper.

Chetty, R., Friedman, J. N., Hendren, N., Stepner, M., and Team, T. O. I. (2020). 'How Did COVID-19 and Stabilization Policies Affect Spending and Employment? A New Real-Time Economic Tracker Based on Private Sector Data'. NBER Working Paper No 27431.

D'Erasmo, P., Mendoza, E., and Zhang, J. (2016). 'What is a Sustainable Public Debt?' Handbook of Macroeconomics, vol. 2, 2493-2597. 
Eichenbaum, M. S., Rebelo, S., and Trabandt, M. (2020). 'The Macroeconomics of Epidemics'. NBER Working Paper No 26882.

Elenev, V., Landvoigt, T., and Van Nieuwerburgh, S. (2020). 'Can the Covid Bailouts Save the Economy?' NBER Working Paper No 27207.

Faria e Castro, M. (2020). 'Fiscal Policy during a Pandemic'. Federal Reserve Bank of St. Louis Working Paper No 2020-006E.

Favero, C. A., Ichino, A., and Rustichini, A. (2020). 'Restarting the Economy While Saving Lives'. CEPR Discussion Paper No 14664.

Fornaro, L. and Wolf, M. (2020). ‘Covid-19 Coronavirus and Macroeconomic Policy'. CEPR Technical Report.

Galasso, V. (2020). 'Covid: Not a Great Equaliser'. Covid Economics, vol. 19, 241-255.

Glover, A., Heathcote, J., Krueger, D., and Ríos-Rull, J. (2020). 'Health versus Wealth: On the Distributional Effects of Controlling a Pandemic'. NBER Working Paper No 27046.

Gourinchas, P.-O., Kalemli-Ozcan, S., Penciakova, V., and Sander, N. (2020). 'COVID-19 and SME Failures'. NBER Working Paper No 27877.

Guerrieri, V., Lorenzoni, G., Straub, L., and Werning, I. (2020). 'Macroeconomic Implications of COVID-19: Can Negative Supply Shocks Cause Demand Shortages?' NBER Working Paper No 26918.

Halpern, N. A. and Tan, K. (2020). 'United States Resource Availability for COVID-19'. Society of Critical Care Medicine Report.

Hur, S. (2020). 'The Distributional Effects of COVID-19 and Optimal Mitigation Policies'. Federal Reserve Bank of Dallas Working Paper.

Jones, C. J., Philippon, T., and Venkateswaran, V. (2020). 'Optimal Mitigation Policies in a Pandemic: Social Distancing and Working from Home'. NBER Working Paper No 26984.

Kaplan, G., Moll, B., and Violante, G. (2020). 'The Great Lockdown and the Big Stimulus: Tracing the Pandemic Possibility Frontier for the U.S.' NBER Working Paper No 27794.

Krueger, D., Uhlig, H., and Xie, T. (2020). 'Macroeconomic Dynamics and Reallocation in an Epidemic'. NBER Working Paper No 27047.

Leiserson, G., McGrew, W., and Kopparam, R. (2019). 'The Distribution of Wealth in the United States and Implications for a Net Worth Tax'. Washington Center for Equitable Growth.

Li, R., Rivers, C., Tan, Q., Murray, M., Toner, E., and Lipsitch, M. (2020). 'Estimated Demand for US Hospital Inpatient and Intensive Care Unit Beds for Patients With COVID-19 Based on Comparisons With Wuhan and Guangzhou, China'. JAMA Network Open, vol. 3, no. 5. 
Mendoza, E., Tesar, L., and Zhang, J. (2014). 'Saving Europe?: The Unpleasant Arithmetic of Fiscal Austerity in Integrated Economies'. NBER Working Paper No 20200.

Mongey, S., Pilossoph, L., and Weinberg, A. (2020). 'Which Workers Bear the Burden of Social Distancing Policies?' NBER Working Paper No 27085.

Palomino, J., Rodríguez, J., and Sebastian, R. (2020). 'Wage inequality and poverty effects of lockdown and social distancing in Europe'. European Economic Review, vol. 129, 103564.

Rampini, A. (2020). 'Sequential Lifting of COVID-19 Interventions with Population Heterogeneity'. NBER Working Paper No 27063. 